\title{
Gut-Lung Dysbiosis Accompanied by Diabetes Mellitus Leads to Pulmonary Fibrotic Change through the NF- $\kappa$ B Signaling Pathway
}

Guang Wang, ${ }^{* \dagger}$ Yu-Xuan Hu, ${ }^{*}$ Mei-Yao He, ${ }^{*}$ Yun-Hai Xie, ${ }^{*}$ Wei Su, ${ }^{*}$ Denglu Long, ${ }^{*}$ Ran Zhao, ${ }^{*}$ Jingyun Wang, ${ }^{\ddagger}$ Chenyang Dai, Haiyang $\mathrm{Li}^{*}{ }^{*}$ Zhen-Peng $\mathrm{Si},{ }^{\ddagger}$ Xin Cheng, ${ }^{*}$ Rui-Man Li, ${ }^{\S}$ Zhijie Li, ${ }^{ף}$ and Xuesong Yang ${ }^{* \dagger}$

From the International Joint Laboratory for Embryonic Development and Prenatal Medicine, * Division of Histology and Embryology, Medical College, and the Key Laboratory for Regenerative Medicine of the Ministry of Education, ${ }^{\dagger}$ Jinan University, Guangzhou; Department of Obstetrics and Gynecology, ${ }^{\ddagger}$ Department of Pediatrics, ${ }^{\S}$ The First Affiliate Hospital of Jinan University, Guangzhou; and the International Ocular Surface Research Center, ${ }^{\mathbb{1}}$ Institute of Ophthalmology, Key Laboratory for Regenerative Medicine of the Ministry of Education, Jinan University, Guangzhou, China

Accepted for publication February 10, 2021.

Address correspondence to Xuesong Yang, Ph.D., Department of Histology and Embryology, School of Medicine, Jinan University, No. 601 Huangpu Rd. W., Guangzhou 510632, China. E-mail: yang xuesong@126.com.

\begin{abstract}
Growing evidence shows that the lungs are an unavoidable target organ of diabetic complications. However, the pathologic mechanisms of diabetic lung injury are still controversial. This study demonstrated the dysbiosis of the gut and lung microbiome, pulmonary alveolar wall thickening, and fibrotic change in streptozotocin-induced diabetic mice and antibiotic-induced gut dysbiosis mice compared with controls. In both animal models, the NF- $\kappa B$ signaling pathway was activated in the lungs. Enhanced pulmonary alveolar well thickening and fibrotic change appeared in the lungs of transgenic mice expressing a constitutively active NF- $\kappa$ B mutant compared with wild type. When lincomycin hydrochloride-induced gut dysbiosis was ameliorated by fecal microbiota transplant, enhanced inflammatory response in the intestine and pulmonary fibrotic change in the lungs were significantly decreased compared with lincomycin hydrochloride-treated mice. Furthermore, the application of fecal microbiota transplant and baicalin could also redress the microbial dysbiosis of the gut and lungs in streptozotocin-induced diabetic mice. Taken together, these data suggest that multiple as yet undefined factors related to microbial dysbiosis of gut and lungs cause pulmonary fibrogenesis associated with diabetes mellitus through an NF- $\kappa$ B signaling pathway. (Am J Pathol 2021, 191: 838-856; https://doi.org/10.1016/j.ajpath.2021.02.019)
\end{abstract}

Diabetes mellitus (DM), also called diabetes, has become a major global public health problem with a considerable increase in the prevalence of DM over the past few decades. ${ }^{1}$ As a metabolic disorder characterized by persistent hyperglycemia, DM consists of autoimmune type 1 diabetes and insulin-resistant type 2 diabetes. $^{2}$ The pathogenesis of DM is deemed to involve a combination of genetic and environmental factors. ${ }^{3,4}$ Poorly controlled diabetes can lead to various complications, such as retinopathy, nephropathy, and neuropathy. ${ }^{5}$ In addition, accumulating evidence reveals that DM can induce inflammatory and fibrotic changes in the lungs, ${ }^{6}$ leading to several abnormalities of the respiratory functions (eg, lung volume, pulmonary diffusing capacity, and control of ventilation). ${ }^{7}$ Although these characteristic pulmonary histologic changes induced by diabetes may be mild, certainly consider the diabetic lung as a target organ in DM, can accelerate the aging process in pulmonary functions. ${ }^{7}$ However, how diabetes causes the inflammatory responses and further develops to lung injury and pulmonary fibrosis is still not well understood.

Supported by National Nature Science Foundation of China grants 31971108 (G.W.) and 31771331 (X.Y.); the Science and Technology Planning Project of Guangdong Province grants 2017A050506029 (X.Y.), 2017A020214015 (G.W.), and 2016B030229002 (X.Y.); Special Funds for the Cultivation of Guangdong College Students' Scientific and Technological Innovation pdjh2019b0066 (Y.-X.H.); and National Innovation and Entrepreneurship Training Program for Undergraduate 201810559028 (Y.X.H.).

G.W., Y.-X.H., and M.-Y.H. contributed equally to this work.

Disclosures: None declared. 
Gut microbiota dysbiosis is closely interrelated with DM in both rodent models and humans, ${ }^{8}$ although the causal relationship between gut microbiota dysbiosis and developing diabetes is still not completely elucidated. Maintaining the health and integrity of the gut microbiome from childhood is crucial to preventing diabetes, despite the unclear mechanism regarding the interaction between gut microbiota and host immunity. ${ }^{8-10}$ The intestinal bacteria colonize immediately after birth, and the diversity and richness of the microbial composition reach their highest complexity in adulthood. ${ }^{11}$ Meanwhile, growing evidence in human and animal models has shown that diabetes and obesity are highly associated with profound gut microbiota dysbiosis. ${ }^{12,13}$

Growing evidence shows a close correlation between the gastrointestinal tract and respiratory tract because chronic gut and lung disorders often share identical characteristics and dysregulation of the microbiome, commonly named the socalled gut-lung axis, the gut microbiome, and respiratory disorder, ${ }^{14}$ indicating the intimate intestinal and pulmonary cross talk in the context of respiratory health and disorders. This has benefited from the identification of microbial organisms in the lungs using microbial 16S RNA gene high-throughput sequencing. ${ }^{15,16}$ The lung microbiome physiologically functions in host immunity like the gut microbiome, and a pathologically imbalanced ecosystem in the lungs could predispose an individual to the onset or development of respiratory disorders, ${ }^{17,18}$ suggesting that qualitative alterations in the lung microbiome might relate to the progression and exacerbation of various pulmonary disorders. Thus, a commensal gut-lung axis (ie, immunologic link between the gut and the lung through the bloodstream) mainly based on immune transmission provides novel insights into the pathogenesis of many chronic pulmonary illnesses. ${ }^{17}$ Therefore, pulmonary inflammation and illness can be derived from the gut microbiota through the gut-lung axis.

NF- $\kappa \mathrm{B}$ plays a crucial role in intestinal immune homeostasis by endorsing mucosal immunity and restricting chronic inflammation. ${ }^{19}$ The activation of NF- $\mathrm{BB}$ signaling depends on the initiation of the signaling cascade via stimulation of cell surface receptors (toll-like receptors) by cytokines/pathogens and the phosphorylation-induced proteasomal degradation of NF- $\kappa \mathrm{B}$ proteins (I $\mathrm{BSs}$ ), which is required for signal transduction to NF- $\kappa B .{ }^{20}$ The initiation, propagation, and development of metabolic diseases, including type 2 diabetes, are largely derived from chronic low-level inflammation in which NF- $\mathrm{BB}$ signaling plays crucial roles in coordinating inflammatory responses and subsequent disease development. ${ }^{21}$ Hence, this study investigated the roles of NF- $\kappa \mathrm{B}$ signaling in mediating the gut-lung microbiota dysbiosis-induced pulmonary fibrotic changes in the context of DM.

\section{Materials and Methods}

\section{Animals and Genetic Manipulations}

The C57BL/6 mice used in this study were obtained from the Institute of Laboratory Animal Science, Jinan University
(Guangzhou, China). NF-кB1(Cys-Ser) knock-in mice were obtained from the Modern Animal Research Center of Nanjing University (Nanjing, China). In the knock-in mice, the usually conservative Cys was replaced by Ser, such that the p50 with this substitution retained a maximum DNAbinding activity, ${ }^{22}$ as shown before. ${ }^{23}$ Multiplex PCR genotyping used four primers to detect the knock-in alleles (primer 1, primer 2, primer 3, and primer 4). The following PCR conditions were used to detect wild-type and NF$\kappa \mathrm{B} 1 \mathrm{C} 59 \mathrm{~S}$ alleles: $94^{\circ} \mathrm{C}$ for 5 minutes; 41 cycles of $94^{\circ} \mathrm{C}$ for 30 seconds, $58^{\circ} \mathrm{C}$ for 30 seconds, and $72^{\circ} \mathrm{C}$ for 45 seconds; and $72^{\circ} \mathrm{C}$ for 5 minutes. Primers were obtained from Sangon Biotech (Shanghai, China), and the sequences are listed in Table 1 . The mice were housed in the animal facility under constant room temperature, $50 \%$ to $60 \%$ humidity, and a 12:12-hour light-dark cycle, and were supplied with standard food pellets and water.

Type 1 DM mouse model was established using streptozotocin (STZ) injection. ${ }^{24-26}$ Briefly, 8-week-old mice were injected with STZ (Sigma, St. Louis, MO; dissolved in $0.01 \mathrm{~mol} / \mathrm{L}$ citrate buffer, $\mathrm{pH} 4.5$ ) at $75 \mathrm{mg} / \mathrm{kg}$ body weight for 3 consecutive days, and diabetes mellitus was defined as a fasting blood glucose level $>288 \mathrm{mg} / \mathrm{dL}(>16 \mathrm{mmol} /$ L). ${ }^{27-31}$ Similarly, an antibiotic treatment mouse model ${ }^{32}$ and lincomycin hydrochloride (LH) treatment mouse model, were established, as described before. ${ }^{33}$

The mice in control group $(n \geq 10)$ were treated with physiological saline solution (containing $0.1 \%$ dimethyl sulfoxide). Mice with DM were randomly divided into three groups: i) physiological saline treatment group $(n \geq 12)$, ii) fecal microbiota transplant (FMT) treatment group $(n \geq 6)$, and iii) baicalin treatment group ( $n \geq 6)$, from at least three different batches. In control and DM groups, the mice were administered with physiological saline solution via an intragastric gavage for 1 week. For the FMT, $200 \mathrm{mg}$ of stool (from normal mouse pellets) was resuspended in $5 \mathrm{~mL}$ of phosphate-buffered saline under anaerobic conditions, vortexed for 3 minutes, and allowed to settle by gravity for 2 minutes. ${ }^{34}$ Each DM mouse in the FMT group was given $200 \mu \mathrm{L}$ of the supernatant by intragastric method every 3 days. ${ }^{32}$ The mice in baicalin group were administered $1 \mathrm{~mL} /$ day of baicalin $(40 \mathrm{mg} / \mathrm{kg}$ body weight) by intragastric gavage for 1 week. Mice with LH-induced microbiota dysbiosis were randomly divided into two groups: FMT treatment group $(n \geq 6)$ and physiological saline treatment group $(n \geq 6)$.

Table 1 Primer of Mouse Genotyping

\begin{tabular}{|c|c|c|}
\hline Genotyping & Primer & Sequence \\
\hline \multirow[t]{2}{*}{$\mathrm{NF}-\kappa \mathrm{B} 1^{\mathrm{C598}}$} & Forward & 5'-CCTGCTTACAACCTGTTTATTC-3' \\
\hline & Reverse & 5'-GCATCGCATTGTCTGAGTAGGTG-3' \\
\hline \multirow[t]{2}{*}{ Wild type } & Forward & 5'-CCTGCTTACAACCTGTTATTC-3' \\
\hline & Reverse & 5'-GTGAGATTGGTTCCAACATCAAC-3' \\
\hline
\end{tabular}


Table 2 PCR Primer

\begin{tabular}{|c|c|c|}
\hline Gene & Primer & Sequence \\
\hline \multirow[t]{2}{*}{ Cd19 } & Forward & 5'-TGGTGGAGGTAGAAGAGGG-3' \\
\hline & Reverse & 5'-GGAAGGGTGTTGACTGGTTA-3' \\
\hline \multirow[t]{2}{*}{$\mathrm{Cd} 20$} & Forward & 5'-TATTCAAACTTCCAAGCCGTAT-3' \\
\hline & Reverse & $5^{\prime}-\mathrm{ATCAGCATCGCCGACAGA}-3^{\prime}$ \\
\hline \multirow[t]{2}{*}{ Cd14 } & Forward & $5^{\prime}-\mathrm{TCTTGAACCTCCGCAACG-3'}$ \\
\hline & Reverse & $5^{\prime}-$ TGGGCAATACTCAGTACCTTGA-3' \\
\hline \multirow[t]{2}{*}{ Cd45 } & Forward & $5^{\prime}-$ CCCGCACTGAGCTGGAAT- $3^{\prime}$ \\
\hline & Reverse & 5'-TGACACCGCTGCTCCTCTT-3' \\
\hline \multirow[t]{2}{*}{ Cd68 } & Forward & 5'-ATCTTGCTAGGACCGCTTAT-3' \\
\hline & Reverse & 5'-GTGGCTGTAGGTGTCATCGT-3' \\
\hline \multirow[t]{2}{*}{$\operatorname{Tgfb} 1$} & Forward & 5'-ACCGCAACAACGCCATCT-3' \\
\hline & Reverse & $5^{\prime}$-GGGCACTGCTTCCCGAAT-3' \\
\hline \multirow[t]{2}{*}{ Il6 } & Forward & $5^{\prime}-\mathrm{TACCACTCCCAACAGACC-3^{ \prime }}$ \\
\hline & Reverse & $5^{\prime}-\mathrm{TTTCCACGATTTCCCAGA-3'}$ \\
\hline \multirow[t]{2}{*}{ Casp8 } & Forward & $5^{\prime}$-СССАGАTTTСТСССТАСА-3' \\
\hline & Reverse & $5^{\prime}-\mathrm{ATCAGCATCGCCGACAGA}-3^{\prime}$ \\
\hline \multirow[t]{2}{*}{ Casp9 } & Forward & 5'-CGGTGGACATTGGTTCTG-3' \\
\hline & Reverse & $5^{\prime}$-CACATTGTTGATGATGAGGC-3' \\
\hline \multirow[t]{2}{*}{ Bcl2 } & Forward & 5'-GCTACCGTCGTGACTTCGC-3' \\
\hline & Reverse & $5^{\prime}-\mathrm{CTCCACCACCGTGGCAAA-3^{ \prime }}$ \\
\hline \multirow[t]{2}{*}{ Bax } & Forward & 5'-GTTGCCCTCTTCTACTTTGC-3' \\
\hline & Reverse & $5^{\prime}-\mathrm{TCCAGTGTCCAGCCCATG} \mathrm{-3^{ \prime }}$ \\
\hline \multirow[t]{2}{*}{ Col1a1 } & Forward & 5'-CGCCATCAAGGTCTACTGC-3' \\
\hline & Reverse & 5'-GAATCCATCGGTCATGCTCT-3' \\
\hline \multirow[t]{2}{*}{ Col3a1 } & Forward & 5'-GGCAGTGATGGGCAACCT-3' \\
\hline & Reverse & $5^{\prime}-$ TGGTGAGCCATTTGAGCC - $3^{\prime}$ \\
\hline \multirow[t]{2}{*}{ P65 } & Forward & $5^{\prime}-\mathrm{GCACCAAGACCGAAGCAA-3^{ \prime }}$ \\
\hline & Reverse & $5^{\prime}-\mathrm{TCCCGTAACCGCGTAGTC}-3^{\prime}$ \\
\hline \multirow[t]{2}{*}{ Nfkbia } & Forward & $5^{\prime}-\mathrm{ACCAACCAGCCAGGAATT}-3^{\prime}$ \\
\hline & Reverse & $5^{\prime}$-CACAGGCAAGATGTAGAGGG-3' \\
\hline \multirow[t]{2}{*}{ P50 } & Forward & 5'-AAATGGGAAACCGTATGA-3' \\
\hline & Reverse & $5^{\prime}$-TAGCCTCGTGTCTTCTGTC-3' \\
\hline
\end{tabular}

All research involving the use of animals in this study was performed in accordance with the procedures of the Ethical Committee for Animal Experimentation, Jinan University.

\section{Morphologic Analysis of the Mouse Lung}

Hematoxylin and eosin (H\&E), Masson, and Sirius red staining were used to observe histologic pulmonary structures and fibrous tissue of 13-week-old mouse lungs from control, STZ-induced DM, and STZ-induced DM with baicalin treatment groups. Following photography, the lungs, spleens, and large intestines were fixed in $4 \%$ paraformaldehyde, dehydrated, embedded in paraffin wax, and serially divided into sections ( $5 \mathrm{~mm}$ thick). For histologic analysis, the sections were dewaxed in xylene, rehydrated, and stained with H\&E, Masson, or Sirius red, ${ }^{35,36}$ and photographed using a fluorescence microscope (IX50; Olympus, Tokyo, Japan) linked to NIS-Elements F3.2 software (Nikon, Tokyo, Japan). The morphometry of the lung tissues is displayed as images, and six sections per mouse with a minimum of five mice in each group were viewed using a $40 \times$ objective $^{37}$ using Image Pro Plus software version 6.0 (Media Cybernetics, Rockville, MD). The volume density of alveolar spaces was measured by dividing the sum of the airspace area by the total area, ${ }^{38}$ and the histopathologic damage score was calculated. ${ }^{25}$

\section{Immunofluorescence Staining}

The mouse lungs and intestines were fixed in $4 \%$ paraformaldehyde, dehydrated, embedded in paraffin wax, and serially divided into sections (5 $\mu \mathrm{m}$ thick). They were incubated with collagen I (Col1; 1:200; ab34710; Abcam, New Territories, Hong Kong), pro-surfactant protein C (SPC) (1:200; ab90716; Abcam), aquaporin-5 (AQP5; 1:200; ab72876; Abcam), $\alpha$-smooth muscle actin ( $\alpha$-SMA; 1:200; ab66050; Abcam), Ki-67 (1:200; AB9260; Merck KGaA, Darmstadt, Germany), proliferating cell nuclear antigen (1:1000; ab29; Abcam), CD3 (1:200; ab5690; Abcam), p65 (1:200; 6956s; Cell Signaling Technology, Danvers, MA), and caveolin-1 (1:200; PA5-17447; Thermo Scientific, Shanghai, China) overnight at $4^{\circ} \mathrm{C}$. For immunohistochemistry, following extensive washing, the sections were incubated in horseradish peroxidase goat anti-rabbit IgG secondary antibody (1:400; 7074S; EarthOx, Millbrae, CA) for 2 hours at room temperature in a dark box and were conjugated to diaminobenzidine (Maixin, Fuzhou, China). After immunostaining, the sections were counterstained with hematoxylin. For immunofluorescence staining, the sections were incubated with the corresponding Alexa Fluor 555 or 488 secondary antibody (1:1000; Invitrogen, Waltham, MA) at room temperature for 2 hours in a dark box. All sections were subsequently counterstained with DAPI (1:1000; Invitrogen) at room temperature for 30 minutes. The sections were photographed using a fluorescence microscope (Olympus BX53) linked to the cellSens Standard 1.9 software (Olympus Corporation, Tokyo, Japan). A minimum of five random images from three samples were assayed per group. The percentages of cells with positive fluorescence from the immunostaining assay were quantified by measuring the area of immunofluorescence signals with Image Pro Plus 7.0 software. Immunofluorescence staining for cells was performed 48 hours later using antibodies against pro-SPC (1:100; ab90716; Abcam). A minimum of eight images per treatment group were assayed. The cells were photographed using an inverted fluorescence microscope (Ti-U; Nikon, Tokyo, Japan) linked to the NISElements F3.2 software.

\section{Cell Line and CCK8 Assay}

MLE-12, a mouse lung epithelial cell line, was obtained from the ATCC (Manassas, VA; CRL-2110). All of the cells were passaged fewer than 6 months after resuscitation and were cultured in compliance with the protocol provided by the ATCC. Sera and media were purchased from Gibco (Gaithersburg, MD). The cells were cultured in a humidified 


\section{Gut microbiota in diabetic mice}

A

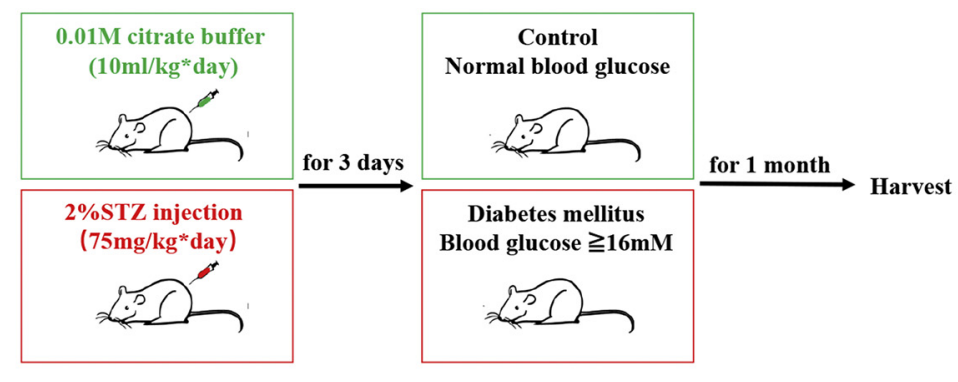

D

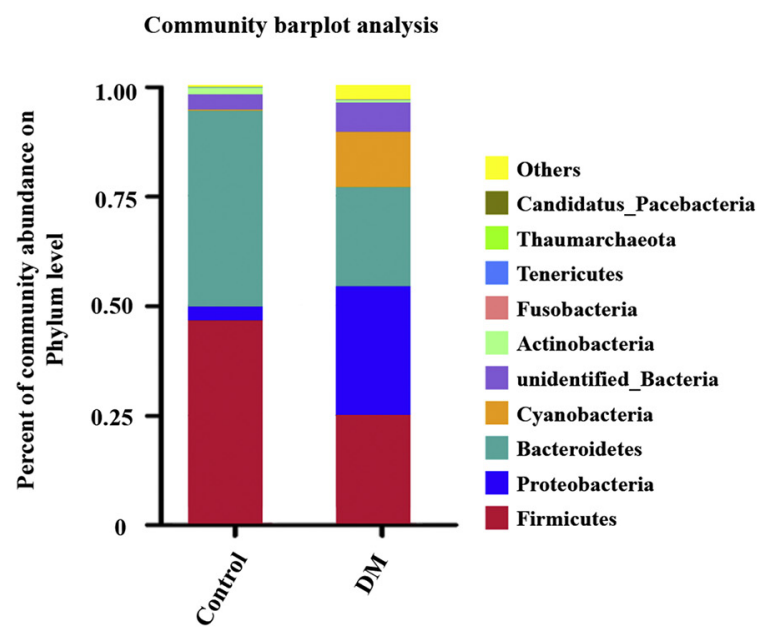

B

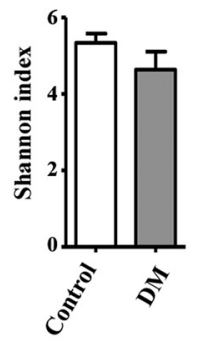

E

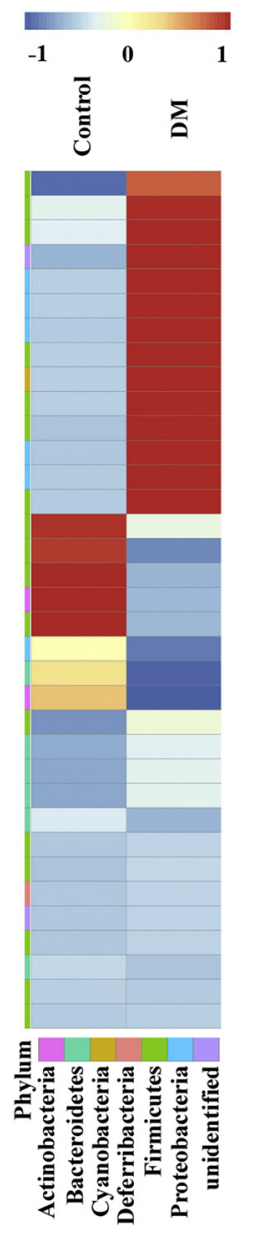

Figure 1 Assessing lung injury in a streptozotocin (STZ)-induced diabetic mouse model of dysbiosis of the gut. A: Illustration showing the production of the control or STZ-induced diabetic mouse model. B and C: Shannon diversity index (B) and first principal component (PC1; C) were calculated in gut dysbiosis. D: Comparing the relative abundances of various bacterial divisions in the gut microbiota. E: A heat map of the differentially expressed genes in the gut microbiota in an RNA-sequencing data set. $n=5$ (C). ${ }^{* * * P}<0.001$. DM, diabetes mellitus.

\section{C}

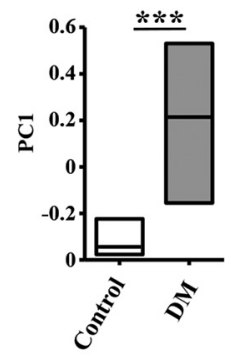

incubator with $5 \% \mathrm{CO}_{2}$ at $37^{\circ} \mathrm{C}$ in 6-well plates $\left(1 \times 10^{6}\right.$ cells/mL) containing Dulbecco's modified Eagle's medium (Gibco) supplemented with $10 \%$ fetal bovine serum (Gibco). Cells were then exposed to $10 \mu \mathrm{g} / \mathrm{mL}$ lipopolysaccharide (LPS) $)^{39,40}$ or $50 \mathrm{mmol} / \mathrm{L}$ D-glucose ${ }^{41,42}$ [high glucose (HG) group; Sigma], whereas blank medium or 50 $\mathrm{mmol} / \mathrm{L}$ mannitol was used as a control, respectively. When needed, $10 \mu \mathrm{mol} / \mathrm{L}$ Bay-11-7082 $2^{42}$ or 2.5 to $10 \mu \mathrm{mol} / \mathrm{L}$ baicalin $^{30,42-44}$ was added to the medium. Cells were harvested 48 hours later for further analysis. Cell viability was assessed using CCK8 assay (cholecystokinin 8). Briefly, $10 \mu \mathrm{L}$ of CCK8 reagent (Dojindo, Kumamoto, Japan) was added to the 96-well plates for 48 hours at $37^{\circ} \mathrm{C}$. The absorbance values were measured at $450 \mathrm{~nm}$ using a Bio-Rad model 450 microplate reader (Bio-Rad, Hercules, CA). The cell viability was indirectly determined by examining the ratio of the absorbance value of baicalin or/ and LPS or glucose-treated cells relative to the control cells. 
A

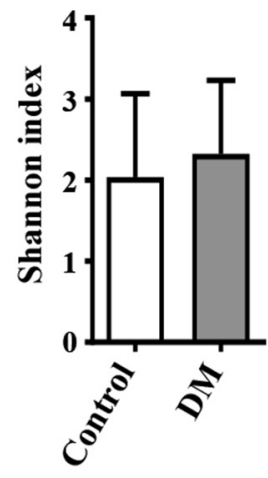

B

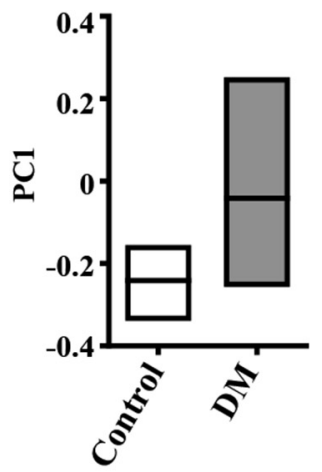

C

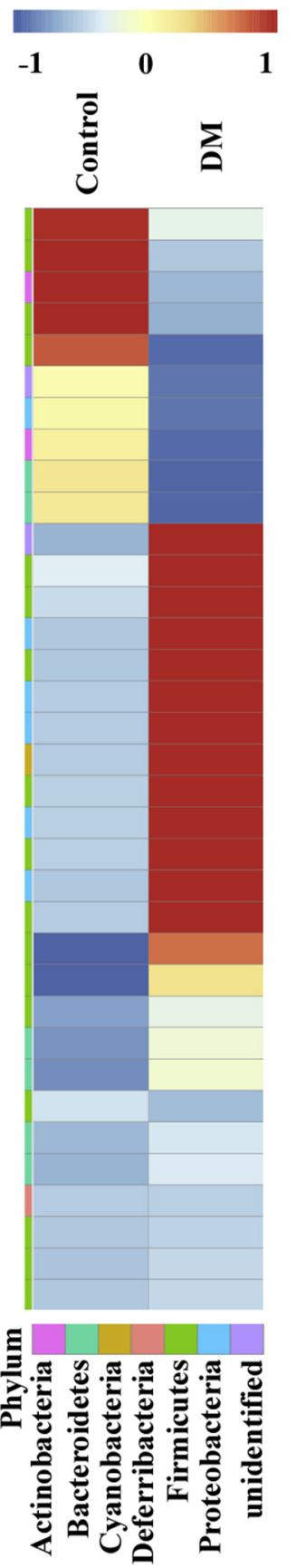

D

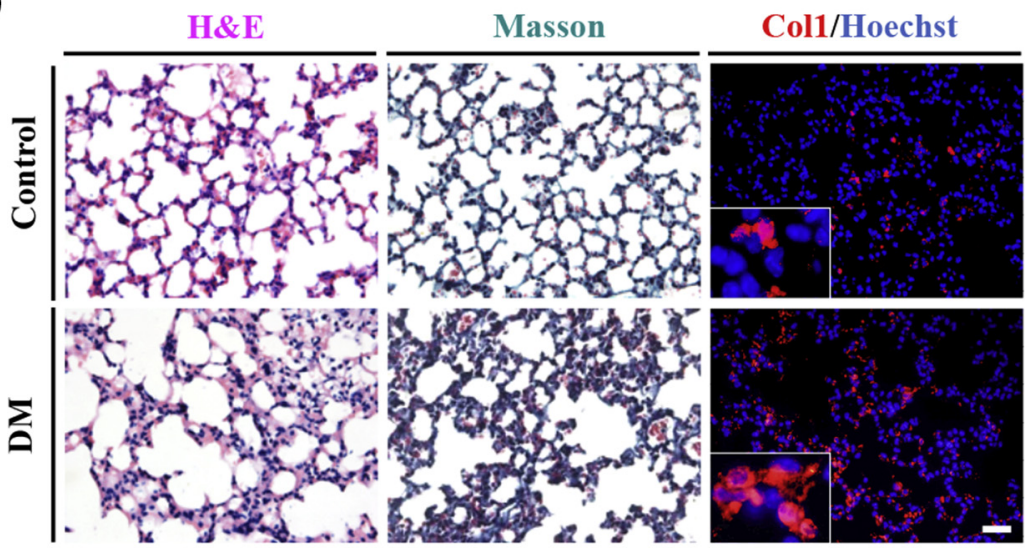

E

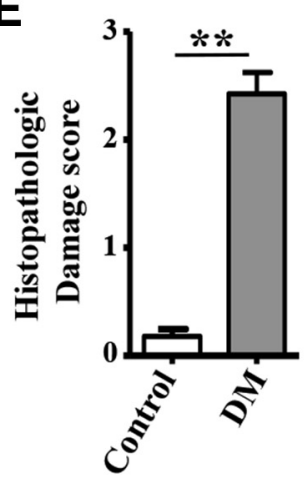

H

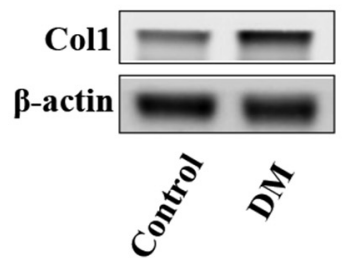

F

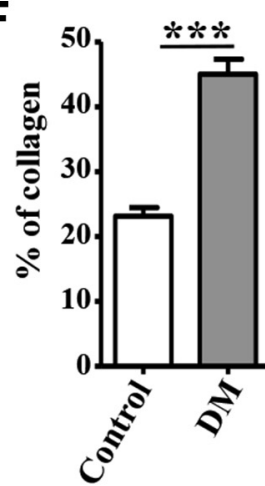

G

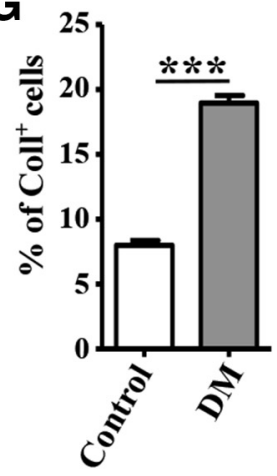

I

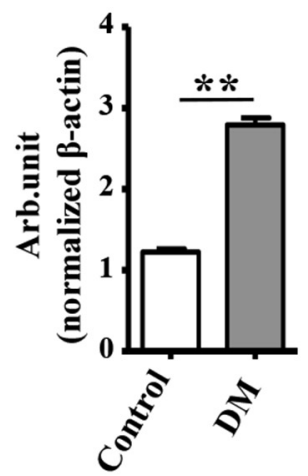

Figure 2 Assessing lung injury in a streptozotocin-induced diabetic mouse model of dysbiosis of lung microbiome. A and B: Shannon diversity index (A) and first principal component (PC1; B) were calculated in lung dysbiosis. C: A heat map of the differentially expressed genes in the lung microbiota in an RNA-sequencing data set. D-G: Hematoxylin and eosin (H\&E), Masson staining, and collagen I (Col1) immunofluorescence staining (Hoechst counterstain) of the transverse sections of mouse lungs from the control and diabetes mellitus (DM) groups. Insets in D show higher magnification. $\mathbf{E}-\mathbf{G}$ : Bar charts showing the histopathologic damage score (E), the percentage of Masson-stained collagen fibers (F), and the percentage of Col1-positive cells in the total cell populations (G). $\mathbf{H}$ and I: Western blot data showing Col1 expression in the mouse lung tissues. $n=5(\mathbf{E}$ and $\mathbf{G}) ; n=6(\mathbf{F}) ; n=3(\mathbf{I}) .{ }^{* *} P<0.01,{ }^{* * *} P<0.001$. Scale bars $=20 \mu \mathrm{m}$ (D)). 


\section{Western Blot Analysis}

Proteins from the mouse lung or MLE-12 cells were isolated from tissue homogenates using a radioimmunoprecipitation assay (Sigma) buffer supplemented with protease and phosphatase inhibitors. Protein concentrations were quantified with the bicinchoninic acid assay. The extracted proteins were separated by $10 \%$ SDS-PAGE and transferred onto a polyvinylidene difluoride membrane (Millipore). The membrane was blocked with 5\% nonfat milk and then incubated with Coll (1:1000; ab34710; Abcam), pro-SPC (1:1000; ab90716; Abcam), AQP5 (1:1000; ab72876; Abcam), $\alpha$-SMA (1:1000; ab66050; Abcam), p65 (1:1000; 6956s; Cell Signaling Technology), IкB $\alpha \quad(1: 1000 ; \quad$ ab109509; Abcam), IL-6 (1:1000; ab233706; Abcam), zonula occludens protein 1 (1:1000; 14667-1-AP; Proteintech, Wuhan, Hubei, China), engagement of the cell death surface receptor $(1: 500 ; 8023 \mathrm{~s}$; Cell Signaling), p53 (1:1000; 2527S; Cell Signaling), P50 (1:1000; bs-1194R; Bioss, Beijing, China), $\beta$-actin (1:3000; 60008-1-1g; Proteintech), and glyceraldehyde-3-phosphate dehydrogenase $(1: 3000$; 60004-1-Ig; Proteintech) in a trisbuffered saline with Tween 20 buffer at $4^{\circ} \mathrm{C}$ overnight. After incubation with the secondary antibody, either horseradish peroxidase goat anti-rabbit IgG (1:3000; 7074S; EarthOx) or horseradish peroxidase goat anti-mouse $\operatorname{IgG}(1: 3000 ; 7076 \mathrm{~S}$; EarthOx), the samples were developed with SuperSignal West Femto Chemiluminescent Substrate (ThermoFisher, Shanghai, China) and the Gel Doc $\mathrm{XR}+$ System (Bio-Rad). Quantity One software version 4.6.2 (Bio-Rad) was used to capture the chemiluminescent signals and analyze the data.

\section{$16 \mathrm{~S}$ rRNA Sequencing Analysis}

The diversity and abundance of the microbiota were analyzed with 16S rRNA gene sequencing. The DNA of the gut microbiota from the stool or lung tissue of the mouse was obtained by the E.Z.N.A. DNA Kit (Omega Bio-tek, Norcross, GA). A DNA sample was sent to the Shanghai Majorbio Bio-Pharm Technology Co., Ltd. (Shanghai, China) for 16S rRNA gene sequencing analysis. 16S rRNA sequence data have been deposited in the Sequence Read Archive (http://www.ncbi.nlm.nih.gov/sra; accession numbers SRP301296, SRP254066, and SRP275674).

\section{RNA Isolation and Quantitative PCR}

Total RNA was extracted from mouse lung using the TRIzol kit (Invitrogen). First-strand cDNA was synthesized to a final volume of $20 \mu \mathrm{L}$ using a SuperScript RIII first-strand kit (Invitrogen). Following reverse transcription, PCR amplification of the cDNA was performed using chickenspecific primers. Primer sequences are provided in Table 2. The PCRs were performed in a Bio-Rad S1000 Thermal cycler (Bio-Rad), as previously described. ${ }^{33}$

\section{Data Analysis}

Statistical analysis was performed using the SPSS Statistics for Windows version 13.0 statistical package program (SPSS Inc, Chicago, IL). Statistical charts were created using the GraphPad Prism 5 software package (GraphPad Software, San Diego, CA). Data are presented as the means \pm SEM in Supplemental Table S1. All data were analyzed using analysis of variance, least significant difference, and Student-Newman-Keuls or the $t$-test. $P<0.05$ was considered statistically significant.

\section{Results}

\section{Pulmonary Fibrotic Change 0ccurrs with Induced Dysbiosis of the Gut and Lung Microbiome}

DM may increase the risk of reduced lung function compared with no DM. To investigate if the dysbiosis of the gut and lung microbiome plays a crucial role in the pathologic process, the current study first determined the gut and lung microbiota in STZ-induced DM mice while assessing the basic structure of the mouse lungs using histochemical approaches (Figure 1). RNA-sequencing data showed a lower Shannon diversity index and higher first principal component values in the DM group compared with the control. Differences in the relative abundance of the gut microbiota phyla (Figure 1), and different hierarchical clustering of bacterial microbiota composition profiles in the heat map (Figure 1) in the DM group compared with the control indicate that DM is associated with the alteration of gut microbiota. Meanwhile, the RNA-sequencing data in the lung microbiota similarly indicated decreased Shannon diversity index and first principal component values in the DM group compared with those in the control. The different hierarchical clustering of the bacterial microbiota composition profiles in the heat map (Figure 2) indicated the presence of lung dysbiosis in STZ-induced diabetic mice.

In mice with microbial dysbiosis of the gut-lung axis, $\mathrm{H} \& \mathrm{E}$ and Masson staining (Figure 2) on the transverse sections of mouse lungs showed a higher histopathologic damage score and collagen synthesis in the DM group compared with that in the control, and Col1 immunofluorescence staining and Western blotting displayed higher expression of Coll in DM mice compared with that in the control (Figure 2). To further study if the observed pulmonary fibrotic changes mentioned above are derived from the dysbiosis of the gut-lung microbiota, this study generated a mouse model of antibiotic-induced gut dysbiosis (Supplemental Figure S1) and found a similar pulmonary fibrotic change because of increased histopathologic damage score and collagen synthesis in the antibiotics group compared with the control, as revealed by H\&E and Masson staining on transverse sections of the lungs (Supplemental Figure S1). 
A

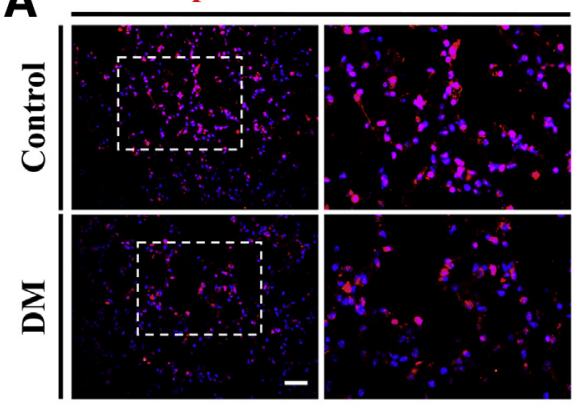

E

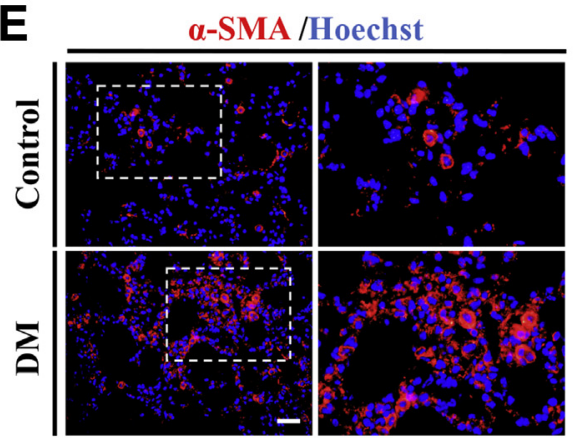

B

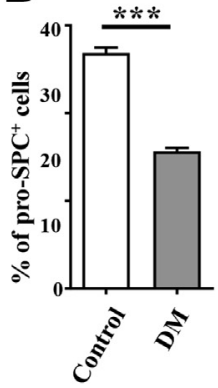

F

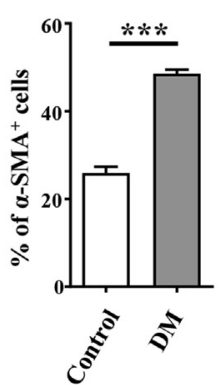

J

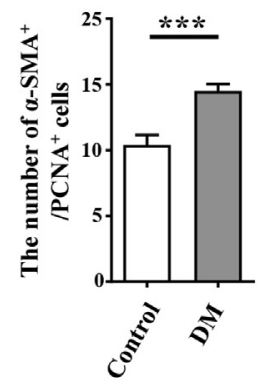

C

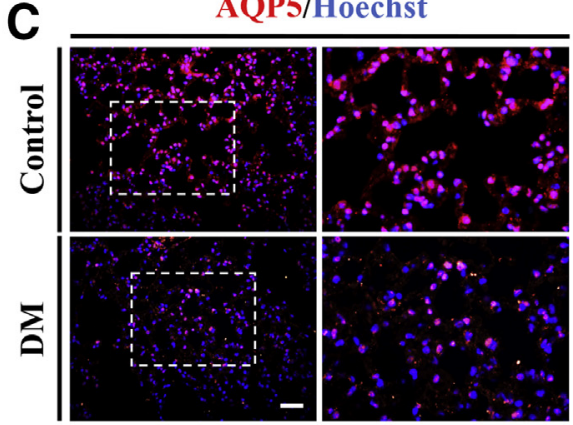

G

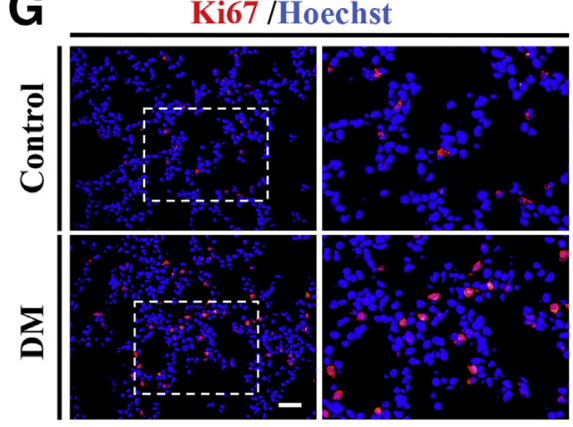

D

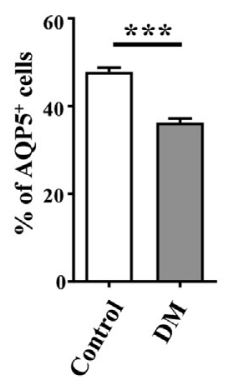

H

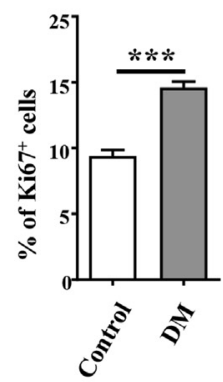

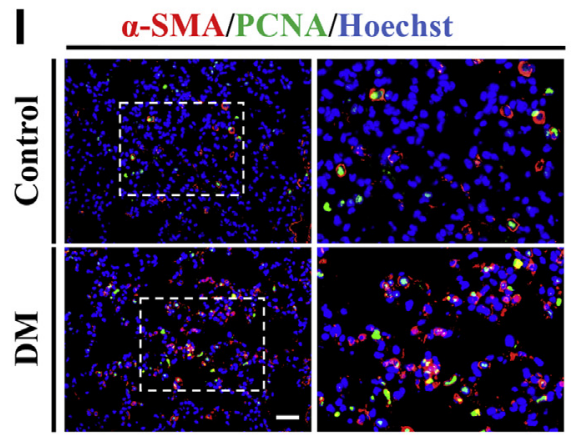
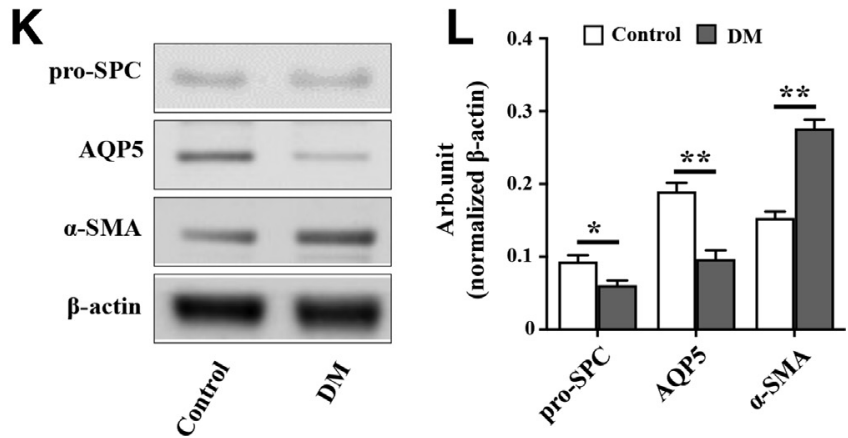

Figure 3 Assessing cell proliferation, differentiation, and fibrotic changes in lung tissue in a streptozotocin-induced diabetic mouse model of dysbiosis of the gut and lung microbiome. A-J: Pro-SPC (A and B), aquaporin-5 (AQP5; C and D), $\alpha$-smooth muscle actin ( $\alpha$-SMA; E and F), Ki-67 (G and $\mathbf{H})$, and $\alpha-S M A /$ proliferating cell nuclear antigen (PCNA; I and $\mathbf{J}$ ) immunofluorescence staining of the transverse sections of mouse lungs from the control and diabetes mellitus (DM) groups. All of the sections were counterstained with Hoechst. A, C, E, G, and I: Boxed areas are shown at higher magnification in the right-side bar charts right columns. The right-side bar charts showing the percentages of pro-SPC-, AQP5-, $\alpha-S M A-$, Ki-67-, and $\alpha$-SMA/PCNA-positive cells in the total cell populations. K: Western blot data showing pro-SPC, AQP5, and $\alpha$-SMA expression in the mouse lung tissues. L: The bar chart shows the ratios of the band arbitrary units of the corresponding gene expression normalized to $\beta$-actin. $n=5$ (B, D, F, $\mathbf{H}$, and $\mathbf{J}) ; n=3(\mathbf{L})$. ${ }^{*} P<0.05,{ }^{*} P<0.01$, and $* * * P<0.001$. Scale bars $=20 \mu \mathrm{m}(\mathbf{A}, \mathbf{C}, \mathbf{E}, \mathbf{G}$, and $\mathbf{I})$. SPC, surfactant protein $\mathbf{C}$.

\section{Cellular Component Alterations of the Pulmonary} Fibrotic Change in Lungs Accompany Dysbiosis of the Gut and Lung Microbiome

To investigate the cellular-biological mechanisms of the pulmonary fibrotic change in gut-lung microbiota dysbiosis induced by DM, immunofluorescence staining and Western blotting using the cell type-specific markers of pulmonary alveolar epithelial and interstitial cells were performed (Figure 3). ${ }^{45-47}$ The results showed that both pro-SPC-positive lung type II alveolar epithelial cells (AECIIs) and AQP5-positive lung type I alveolar epithelial cells significantly decreased in DM mice compared with those in controls (Figure 3). On the other hand, the numbers of $\alpha$-SMA-positive lung interstitial cells (Figure 3) and the percentage of the Ki-67-positive total lung proliferating cells (Figure 3) in DM mice were significantly increased in comparison to the controls along with the proportion of $\alpha$-SMA/proliferating cell nuclear antigen double-positive proliferating lung interstitial cells (Figure 3). Semiquantitative Western blot data verified the decreased expression of AQP5 and pro-SPC along with the increased expression of $\alpha$-SMA in DM mouse lung tissues compared with controls (Figure 3, K and L, and 

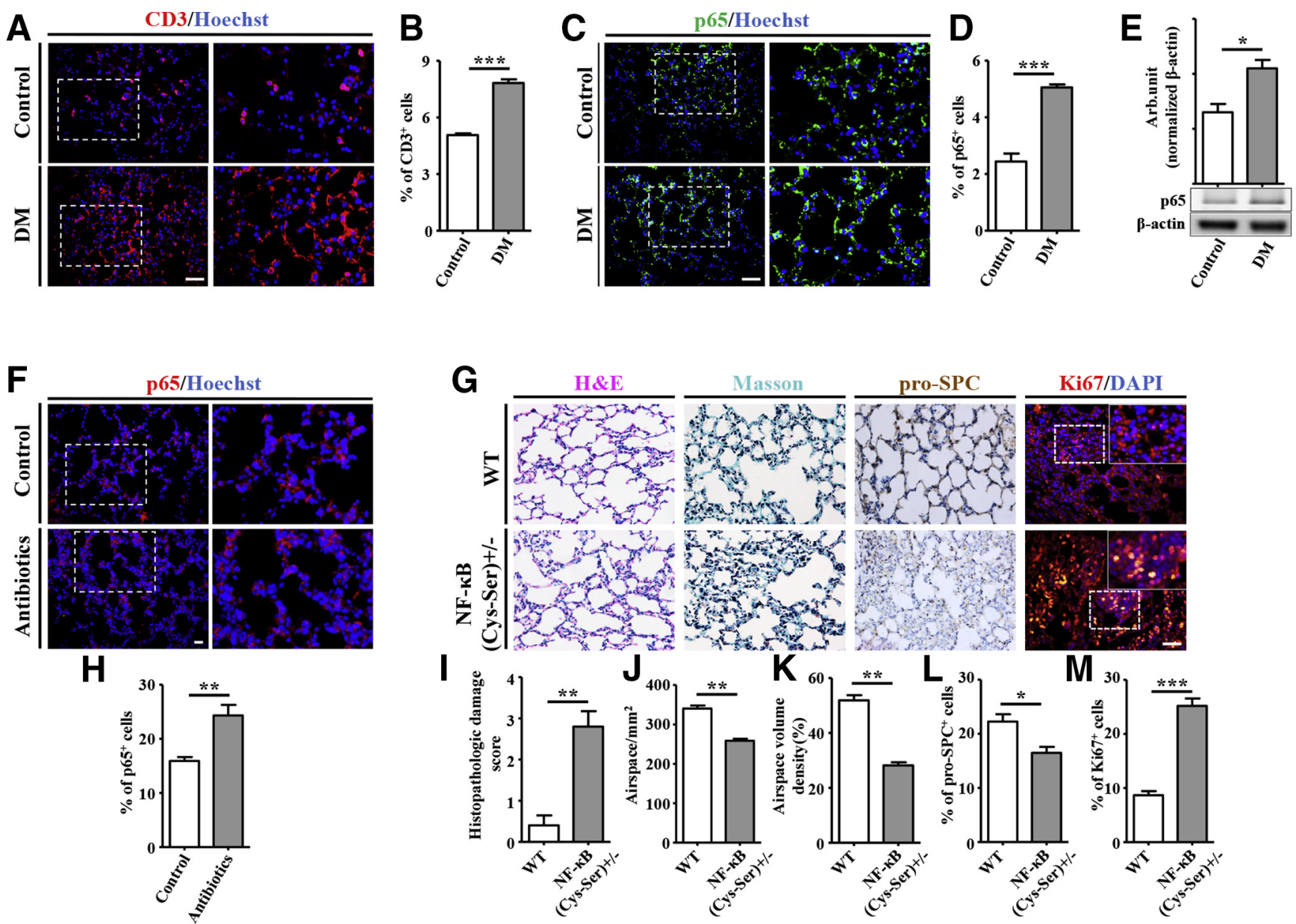

Figure 4 Assessing inflammation, NF- $\kappa B$ signaling, and lung injury in mice with streptozotocin-induced diabetes mellitus (DM) and antibiotic-induced gut dysbiosis. A-D: CD3 (A and $\mathbf{B})$ and p65 (C and $\mathbf{D})$ immunofluorescence staining on the transverse sections of mouse lungs from the control and DM groups. All of the sections were counterstained with Hoechst. The right-side bar charts show the percentages of CD3-and p65-positive cells in the total cell populations. E: Western blot data showing p65 expression in the mouse lung tissues. F: The p65 immunofluorescence staining on the transverse sections of mouse lungs from control and antibiotic-treated mice. All of the sections were counterstained with Hoechst. G: Hematoxylin and eosin (H\&E), Masson staining, prosurfactant protein $C$ cytochemical staining, and $\mathrm{Ki}-67$ immunofluorescence staining (DAPI counterstain) on the transverse right-side bar charts sections of mouse lungs from wild-type and NF- $\kappa B$ (Cys-Ser) ${ }^{+/-}$mice. A, C, and F: Boxed areas are shown at higher magnification in the right columns. G: Boxed areas are shown at higher magnification in the insets. H: The percentages of p65-positive cells in the total cell populations. I-M: The corresponding bar charts show the histopathologic damage scores (I), airspace size per millimeter $(\mathbf{J})$, percentage of airspace volume density (K), and pro-surfactant protein $\mathbf{C}-$ and Ki67-positive cells in the total cell populations ( $\mathbf{L}$ and $\mathbf{M}) . n=5$ (B, D, G, and $\mathbf{I}-\mathbf{M}) ; n=3(\mathbf{E}) .{ }^{*} P<0.05,{ }^{*} P<0.01$, and ${ }^{* * *} P<0.001$. Scale bars $=40$ $\mu \mathrm{m}(\mathbf{A}, \mathbf{C}, \mathbf{F}$, and $\mathbf{G})$. WT, wild type.

Supplemental Figure S2). Next, to determine whether or not the aforementioned pulmonary fibrosis was derived from the direct impact of DM, immunofluorescence staining was performed against these specific markers in mice with antibiotic-induced gut dysbiosis. proSPC-positive type II alveolar epithelial cells were significantly reduced, AQP5-positive AECIs remained relatively stable, and proliferating cell nuclear antigen- or Ki-67-positive pulmonary cell proliferation was significantly enhanced in the lung tissues of the mice with antibiotic-induced gut dysbiosis compared with those in controls (Supplemental Figure S3), implying that the pulmonary fibrotic change phenotypes are associated with the dysbiosis of the gut and lung microbiome.
The NF- $\kappa$ B Pathway Plays a Crucial Role in the Pulmonary Fibrotic Change Occurring with the Dysbiosis of the Gut and Lung Microbiome

Inflammation is a critical factor in the induction of pulmonary fibrosis. ${ }^{48}$ First, markers that help to distinguish leukocyte and macrophage were used Immunofluorescence staining showed that the expression of CD3 (T-cell marker) in STZ-induced diabetic mice was significantly increased in comparison to that in controls (Figure 4). Quantitative PCR results showed that the expressions of CD14 (macrophage marker), CD19 (B-lymphocyte marker), CD20 (macrophage marker), CD45 (lymphocyte common antigen), and CD68 (macrophages and other 
A

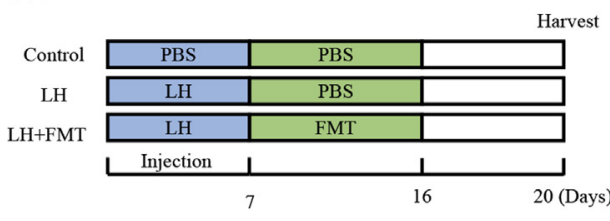

D
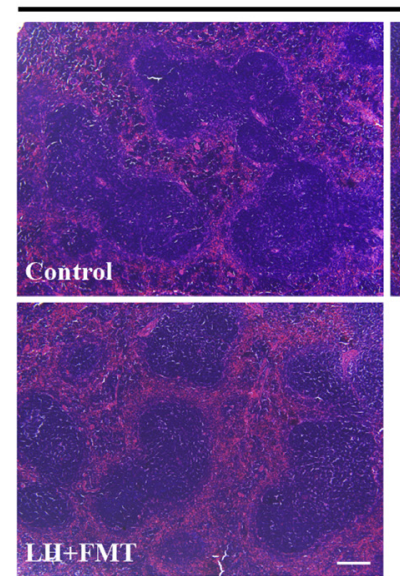

$\mathbf{F}$
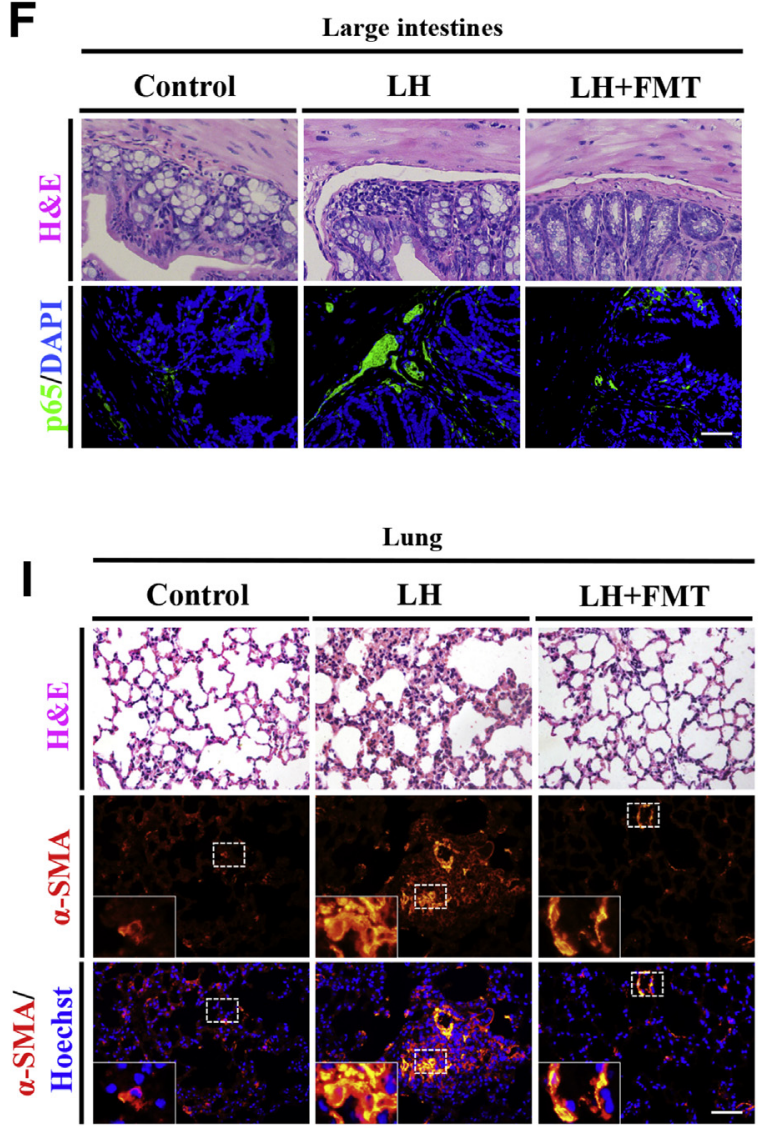

B C

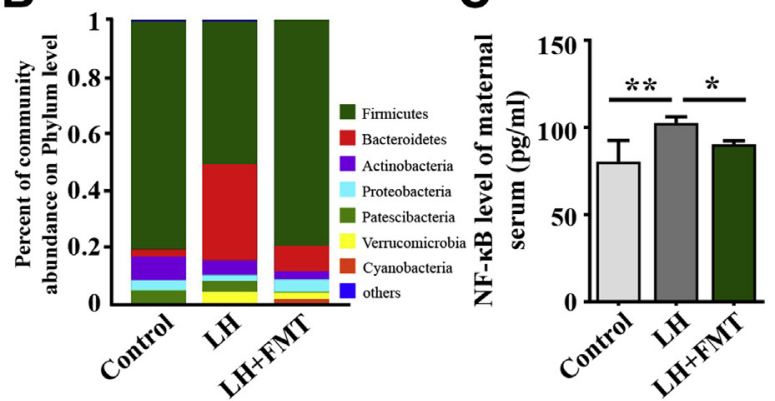

E

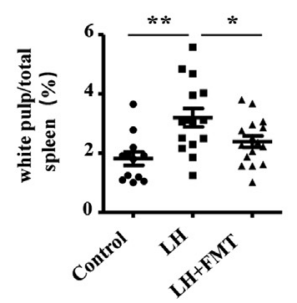

G
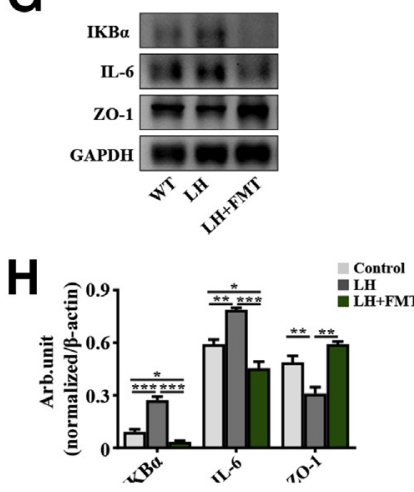

J

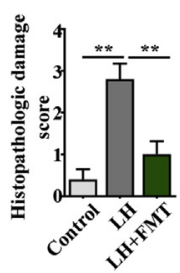

K

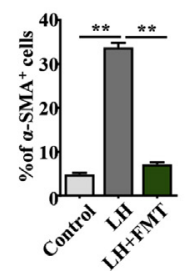


mononuclear phagocytes) were significantly increased in STZ-induced diabetic mice (Supplemental Figure S4). The activation of the NF- $\kappa \mathrm{B}$ signaling pathway is thought to promote pulmonary inflammation and fibrosis. ${ }^{49}$ Hence, here, marker gene expression ${ }^{50}$ of the NF- $\kappa \mathrm{B}$ pathway in mice with dysbiosis of the gut and lung microbiome induced by either STZ or antibiotics as well as the transgenic activation of NF- $\kappa \mathrm{B}$ was determined (Figure 4). The results from immunofluorescence staining and Western blot study showed that p65 expression in STZ-induced diabetic mice (Figure 4, C-E), and in the lungs of mice with antibiotic-induced dysbiosis (Figure 4) was significantly increased in comparison to that in controls. Furthermore, H\&E and Masson staining showed significantly increased histopathologic damage scores/collagen synthesis and significantly decreased airspace size/volume density in the lungs of transgenic mice expressing a constitutively active NF- $\kappa \mathrm{B}$ mutant compared with controls. pro-SPC and Ki-67 immunochemical staining demonstrated that there were lower proportions of proSPC-positive AECIIs and higher proportions of pulmonary cell proliferation in the lungs of transgenic mice with an activated NF- $\kappa \mathrm{B}$ signaling pathway (Figure 4). In addition, the addition of the I $\mathrm{KB} \alpha$ phosphorylation inhibitor Bay-11-7082 rescued the reduction in proSPC-positive AECIIs induced by LPS exposure in mouse lung epithelial (MLE-12) cells in vitro (Supplemental Figure S5).

FMT was performed to further verify the causal relation between the activation of the NF- $\kappa \mathrm{B}$ signaling pathway and gut microbiota dysbiosis. Results indicate, that LH-induced dysbacteriosis was at least partially restored on FM (Figure 5), and the LH-enhanced NF- $\kappa \mathrm{B}$ signaling was significantly lowered by FMT (Figure 5). There was a drop in the proportion of LH-induced increase in mouse splenic white pulp (Figure 5), and the expression of p65 in the large intestines of LH-induced mice was increased compared with the control or FMT (Figure 5). H\&E and $\alpha$-SMA immunostaining showed that FMT resulted in a significantly lower histopathologic damage score and $\alpha$-SMA-positive interstitial cell proportion in the mouse lungs compared with the LH-treated group (Figure 5). These data confirm the importance of the NF- $\mathrm{B}$ signaling pathway in promoting the pulmonary fibrotic change induced by the dysbiosis of the gut and lung microbiome.

\section{FMT Reversed the Pulmonary Fibrotic Change through Inhibition of Apoptosis and Inflammation in STZ- Induced Diabetic Mice}

FMT was performed to further verify the causal relation between the gut microbiota dysbiosis and pulmonary fibrotic change in STZ-induced diabetic mice (Figure 6). There was no significant difference of the blood glucose between the FMT group and the DM group in 24 days, indicating that the FMT failed to reduce the blood glucose of mice (Figure 6). The gut microbiota and lung dysbiosis in STZ-induced mice could be restored to a certain extent, as indicated by the Shannon index, first principal component values, relative abundance of the lung microbiota phyla, and RNA-seq data's heat map (Figure 6). Moreover, FMT reversed the decreased expression of pro-SPC in STZ-induced diabetic mice (Figure 7). Apoptosis may contribute to the pathogenesis of pulmonary fibrosis. ${ }^{51}$ Therefore, the key apoptosis-related genes were studied using Western blot analysis and quantitative PCR. The results suggested that FMT reversed the increased expression of engagement of the cell death surface receptor, p53, Casp8, Casp9, Bcl2, and Bax in STZ-induced diabetic mice (Figure 7). Sirius red staining to stain for collagen can be used to distinguish between type 1 (red or yellow) and type 3 (green) collagens under polarized light. FMT reversed the increased expressions of total collagen, collagen type 1 , and collagen type 3 in STZ-induced diabetic mice (Figure 7). This result was further confirmed by the quantitative PCR (Figure 7). Transforming growth factor (TGF)- $\beta$ is extensively involved in the development of pulmonary fibrosis. ${ }^{52}$ FMT reversed the increased $T g f b l$ expression in diabetic mice as indicated by PCR (Figure 7). Moreover, p65 immunostaining (Figure 7), p65 and p50 Western blot analysis (Figure 7), and p65, Nfkbia, and p50 quantitative PCR results (Figure 7) show that FMT significantly resulted in a lower NF- $\kappa B$ signaling activity compared with that in the STZ-

\footnotetext{
Figure 5 Assessing inflammation and NF- $\mathrm{KB}$ signaling in mice while successfully correcting gut dysbiosis using the fecal microbiota transplant (FMT) approach. A: The sketch illustrates the mouse model of FMT-treated gut microbiota dysbiosis, and white boxes illustrate that there was no specific treatment during 16 to 20 days. B and C: The bar chart shows the relative abundances of various bacterial divisions in gut microbiota following FMT implementation (B) and the determination of the NF- $\mathrm{KB}$ level in maternal serum in the control, lincomycin hydrochloride (LH)-treated, and LH + FMT groups using the enzymelinked immunosorbent assay approach (C). D: Hematoxylin and eosin (H\&E) staining was implemented on the transverse sections of mouse spleens in the control, LH-treated, and LH + FMT groups. E: The scatter diagram shows the ratios of splenic white pulp in the total view. F: H\&E staining and p65 immunofluorescence staining (DAPI counterstain) were implemented on the transverse sections of mouse large intestine in the control, LH-treated, and LH + FMT groups. G and H: Western blot data showing I $\mathrm{B} \alpha \boldsymbol{\alpha}$, IL-6, and zonula occludens protein 1 (Z0-1) expression in the mouse large intestine tissues. The bar chart shows the ratios of the band arbitrary units of the corresponding gene expression normalized to glyceraldehyde-3-phosphate dehydrogenase (GAPDH). I: H\&E staining and $\alpha$-smooth muscle actin ( $\alpha$-SMA) immunofluorescence staining (Hoechst counterstain) were implemented on the transverse sections of mouse lungs in the control, LH-treated, and LH + FMT groups. Boxed areas are shown at higher magnification in the insets. J and K: The corresponding bar charts show the histopathologic damage scores $(\mathbf{J})$ and $\alpha$-SMA-positive cells in the total cell populations (K). $n=6$ for control and LH (C); $n=5$ for LH + FMT (C); $n=12$ for control (E); $n=15$ for LH (E); $n=16$ for LH + FMT (E); $n=3(\mathbf{H}) ; n=5(\mathbf{J}$ and $\mathbf{K}) .{ }^{*} P<0.05, * * P<0.01$, and ${ }^{* * * P}<0.001$. Scale bars: $150 \mu \mathrm{m}$ (D); $40 \mu \mathrm{m}$ (F); $20 \mu \mathrm{m}$ (I). PBS, phosphate-buffered saline.
} 
A

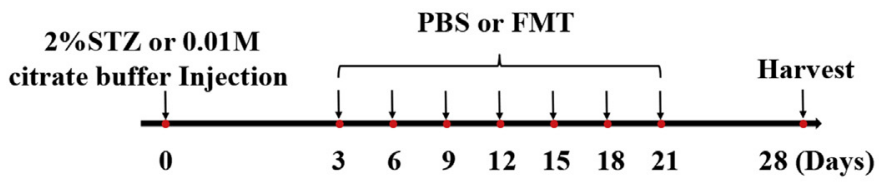

B

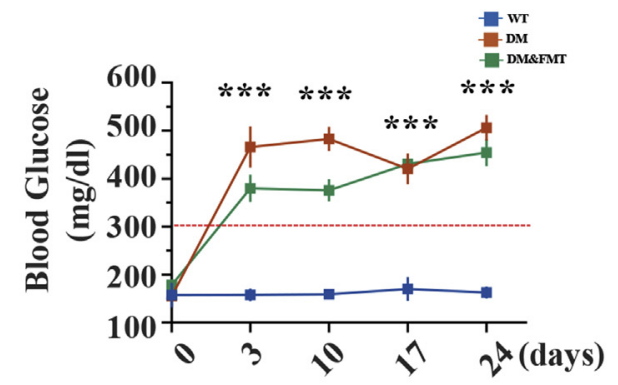

C

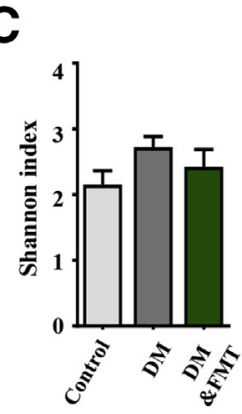

G

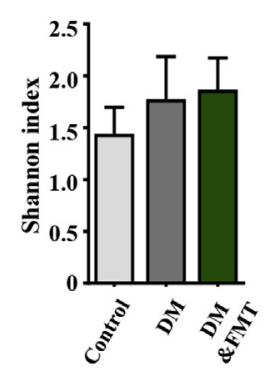

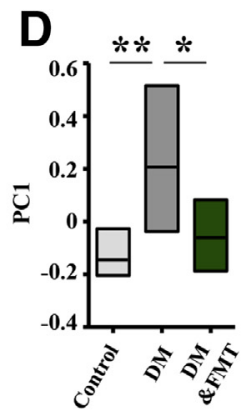

H

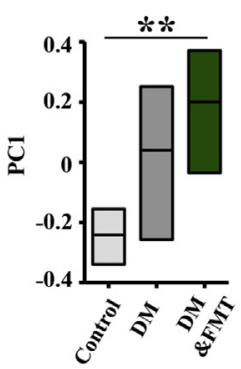

E

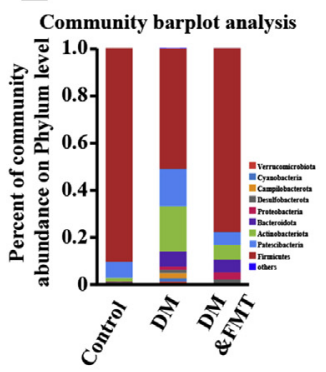

I

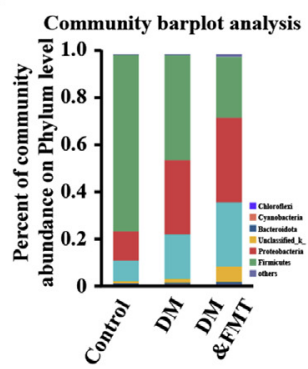

F

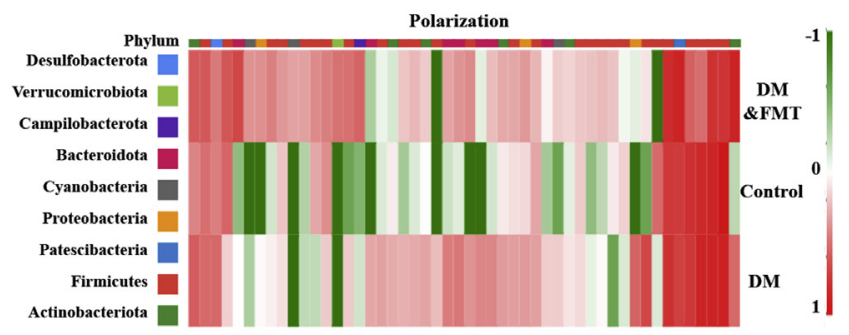

J

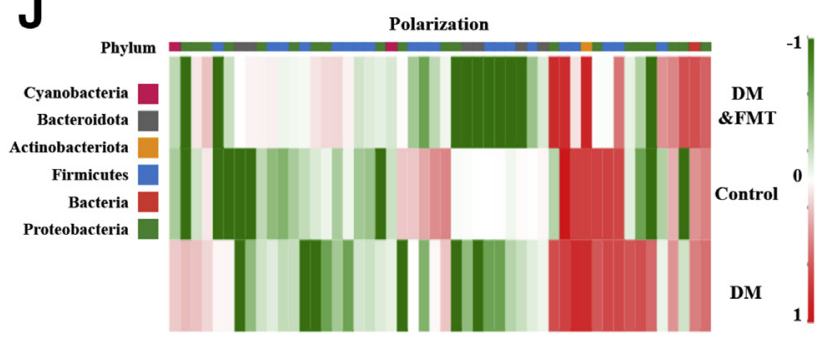

Figure 6 Assessing microbiome in diabetic mice while successfully correcting gut dysbiosis using the fecal microbiota transplant (FMT) approach. A: The sketch illustrates the mouse model of FMT-treated gut microbiota dysbiosis. B: Graph shows the blood glucose of three groups. B: The dashed red line indicates blood glucose of $300 \mathrm{mg} / \mathrm{dL}$. C and D: Shannon diversity index (C) and first principal component (PC1; D) were calculated in gut dysbiosis. E and F: The bar chart showing the comparison of the relative abundances of various bacterial divisions (E) and heat map of the differentially expressed genes in the gut microbiota in the RNA-sequencing data set (F) from the control, diabetes mellitus (DM), and FMT groups. $\mathbf{G}$ and $\mathbf{H}$ : Shannon diversity index (G) and PC1 (H) were calculated in lung dysbiosis. I and J: The bar chart showing the comparison of the relative abundances of various bacterial divisions (I) and heat map of the differentially expressed genes in the lung microbiota in an RNA-sequencing data set (J) from the control, DM, and FMT groups. $n=6$ for control, LH, and LH + FMT (B-D); $n=4$ for control, LH, and LH + FMT (G and $\mathbf{H}) .{ }^{*} P<0.05,{ }^{*} P<0.01$, and ${ }^{* *} P<0.001$. PBS, phosphate-buffered saline; STZ, streptozotocin; WT, wild type.

induced diabetic mice. Quantitative PCR results showed that the expressions of leukocyte and macrophage markers Cd14, Cd19, Cd20, Cd45, and Cd68 in STZ-induced diabetic mice were significantly decreased following FMT treatment (Supplemental Figure S4). These data indicate that FMT in diabetic mice can alleviate STZinduced pulmonary fibrosis through anti-apoptosis and anti-inflammation.

Microbiome Restoration with Baicalin Can Reverse the Activation of NF- $\kappa$ B Signaling and Pulmonary Fibrotic Change in STZ-Induced Diabetic Mice

MLE12 cells were treated with LPS, HG, and/or 2.5, 5, and $10 \mu \mathrm{mol} / \mathrm{L}$ baicalin, an anti-oxidation, anti-inflammation, and anti-apoptosis agent. ${ }^{53}$ CCK8 was used to detect cell proliferation and viability. MLE12 proliferation and viability decreased in the presence of LPS and HG after 48 hours of incubation, and increased with baicalin in a dosedependent manner. Western blot analysis and quantitative PCR results showed baicalin alleviated the expression of p53, p65, Tgfbl, Il6, Casp8, Casp9, and Bcl2 caused by HG or LPS in a dose-dependent manner (Supplemental Figure S6). These results confirm that baicalin can directly protect lung epithelial cells through anti-apoptosis and antiinflammation properties in vitro.

To investigate whether baicalin can reverse gut-lung dysbiosis-induced pulmonary fibrotic change in STZinduced diabetic mice, the mice were administered baicalin orally. The lung microbiota dysbiosis in STZ-induced 
mice was effectively restored by baicalin, as indicated by the RNA-sequencing data's heat map (Figure 8) and the relative abundances of the lung microbiota phyla (Figure 8). The indicators of pulmonary fibrosis (histopathologic damage scores/collagen synthesis and airspace size/volume density) induced by STZ-induced DM were significantly reversed by the oral administration of baicalin (Figure 8). Moreover, baicalin administration also reversed the increased expression of CD3, p65, Col1, and $\alpha$-SMA (Figure 9), as well as up-regulated the decreased expression of pro-SPC and AQP5 in STZ-induced diabetic mice (Figure 9). The p65, pro-SPC, AQP5, Col1, and $\alpha$-SMA Western blot (Figure 8) and the $p 65, I \kappa B \alpha$, and $p 50$ quantitative PCR results (Figure 7) showed that baicalin significantly reversed the pulmonary fibrotic change though and NF- $\kappa \mathrm{B}$ signaling. Quantitative PCR results showed that the expressions of leukocyte and macrophage markers Cd14, Cd19, Cd20, Cd45, and Cd68 were significantly decreased following baicalin treatment in STZ-induced diabetic mice (Supplemental Figure S4). Caveolin-1, which inhibits $\alpha$-SMA, fibronectin, and Coll production, is a critical regulator of lung fibrosis in idiopathic pulmonary fibrosis. ${ }^{54}$ The current study verified that baicalin administration reversed the decreased expression of caveolin-1 in STZ-induced diabetic mice (Supplemental Figure S7). These data suggest that the dysbiosis of gutlung microbiota enhances the risk of pulmonary fibrotic change through activation of the NF- $\kappa \mathrm{B}$ signaling pathway.

\section{Discussion}

Poorly controlled diabetes may cause permanent and profound damage to multiple organs, such as the eyes, liver, brain, kidneys, and heart. Even though the lungs are not one of the most commonly mentioned target organs of diabetes, there is no doubt that the lungs are an unavoidable target of diabetic complications. ${ }^{55}$ Vracko et $\mathrm{al}^{56}$ found that the thickness of alveolar epithelial cells and capillary basal layer in diabetic patients increased in comparison with the peers, but the degree of thickening was not significantly correlated with the age or duration of diabetic patients. However, Weynand et $\mathrm{al}^{57}$ reported that alveolar epithelial cell basal layer, capillary endothelial cell basal layer, and capillary basal layer in the lungs of diabetic patients were significantly increased compared with those in the control group. Matsubara $^{58}$ showed that diabetes mellitus caused an increase in the degree of pulmonary fibrosis of diabetic patients in Japan. The study of Fariña et $\mathrm{al}^{59}$ indicated that diabetes mellitus caused lung inflammatory cell infiltration, excessive deposition of collagen and extracellular matrix, and damage to the basement membrane of the alveolarcapillary barrier, which further led to a particular form of nodular fibrosis. In the light of above-mentioned findings, diabetes mellitus appears to exploit an adverse effect on the pulmonary function, which reflects in progressively causing histopathologic changes in lung and eventually resulting in pulmonary dysfunction. Growing evidence from clinical and experimental data implies that diabetes induces chronic inflammatory and pulmonary fibrotic changes through TGF$\beta$-activated epithelial-mesenchymal transition, ${ }^{6,7}$ which suggests that lung injury could also be one of the long-term implications of diabetes. The pathologic changes at the tissue level induced by diabetes mellitus mainly consist of inflammation and fibrosis, which are initiated by pathogen stimulation and subsequently excessive deposition of extracellular matrix components. ${ }^{60,61}$ However, the underlying pathologic mechanisms are not yet well understood.

The intestinal tract has the largest collection of bacteria in the human body. Under physiological conditions, the intestinal flora and its metabolites form intestinal microecology and maintain dynamic balance, ${ }^{62}$ affecting the human body's nutritional metabolism, immune regulation, and antibacterial processes. ${ }^{63,64}$ Intestinal microecological imbalance, which has been associated with chronic lung diseases, such as asthma, chronic obstructive pulmonary disease, and cystic fibrosis, also shows the symptoms of intestinal diseases. ${ }^{65-67}$ In addition, some respiratory virus infections are often accompanied by intestinal diseases. ${ }^{68,69}$ Studies have shown that the intestinal flora may induce inflammation and oxidative stress by regulating the toll-like receptor $4 / \mathrm{NF}-\kappa \mathrm{B}$ signaling pathway, thereby protecting LPS-induced acute lung injury immunity. Some probiotics can prevent lung diseases through the gut-lung axis, pointing to an interaction between the intestine and the lungs. The destruction of the intestinal flora causes bacterial translocation, activates the lung immune response, induces the production of a large number of inflammatory factors, triggers oxidative stress, and subsequently damages the lung tissue. $^{70}$ FMT may reduce the oxidative damage and enhance the antioxidant capacity. Therefore, the imbalance of the intestinal flora increases the immune response to the bacteria in the lungs through the gut-lung axis and activates the oxidative stress in the lungs. ${ }^{71}$ This study assumed that the activation of the NF- $\kappa \mathrm{B}$ signaling pathway in response to the dysbiosis of the microbiome accompanied by DM plays an important role in diabetes-induced lung injury through the gut-lung axis.

To verify the validity of the aforementioned hypothesis, an STZ-induced diabetic mouse model was first established in which a dysbiosis of both the gut and lung microbiota was found. The neutrophil infiltration/aggregation, alveolar wall thickening, and excessive production of alveolar interstitial collagen were manifested in diabetic mice, which is consistent with previous reports on diabetic lung injury. ${ }^{72,73}$ Likewise, a similar phenotype was observed in the mice with antibiotic-induced gut dysbiosis. It is worthwhile to note that the heat maps between STZ-induced diabetic mice and antibiotic-induced gut dysbiosis are similar. For example, Lachnospiraceae, Helicobacter, Bacteroides, Providencia, and Pseudomonas were all reduced. ${ }^{32}$ AECII cells significantly decreased in both models, 


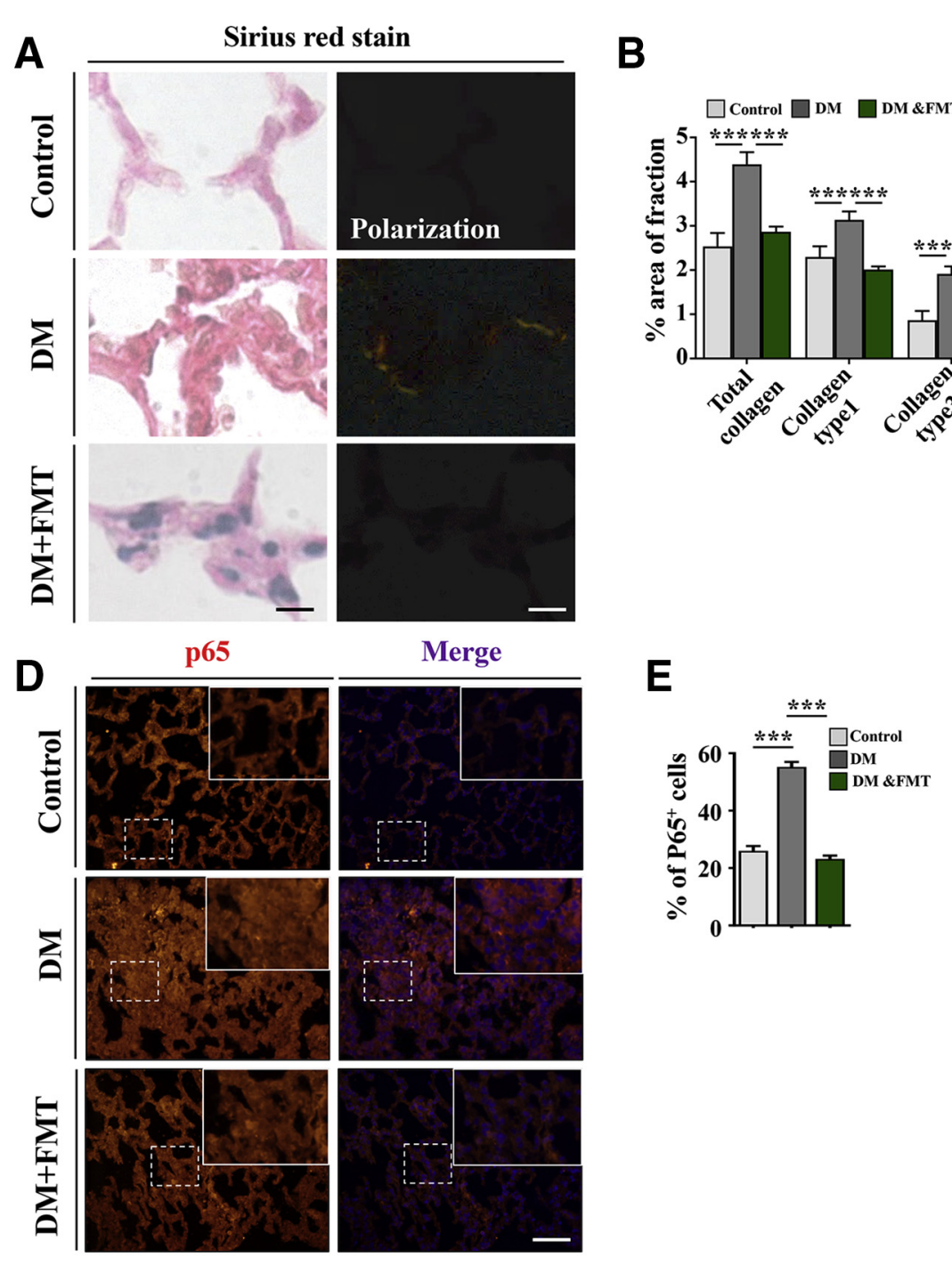

F

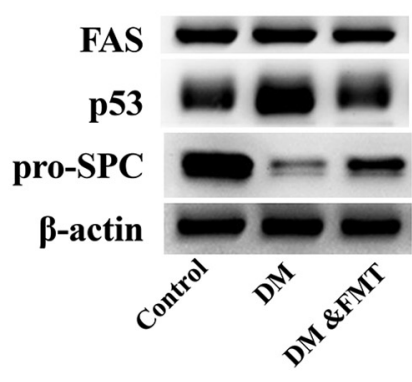

I

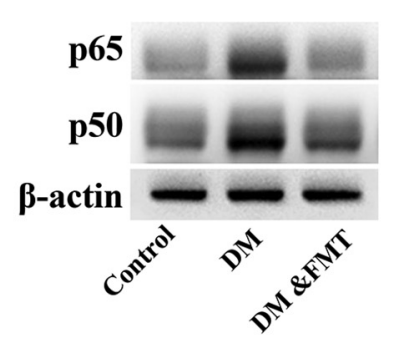

G

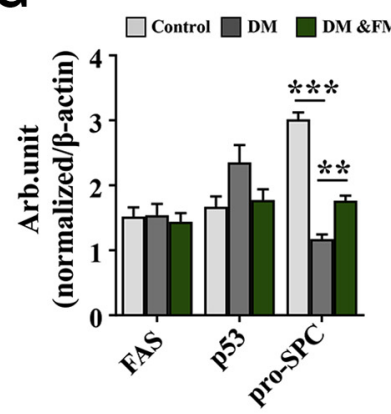

J

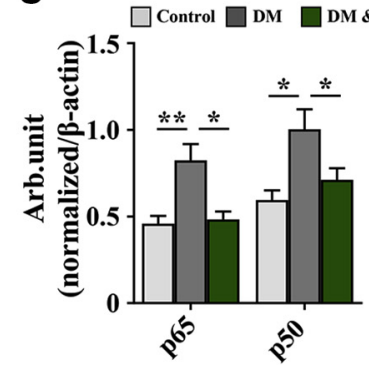

C

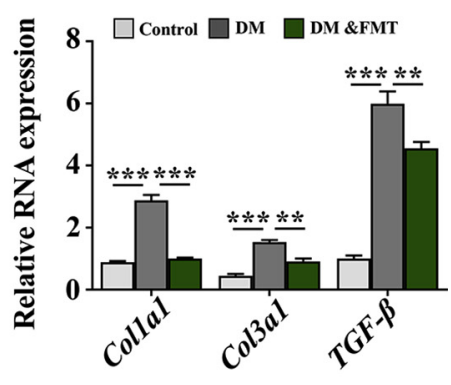

H

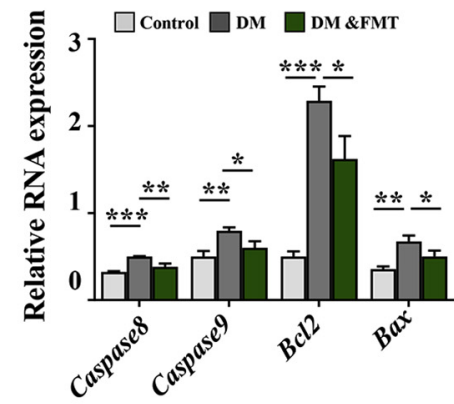

K

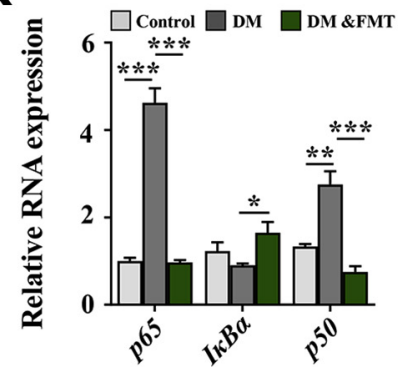



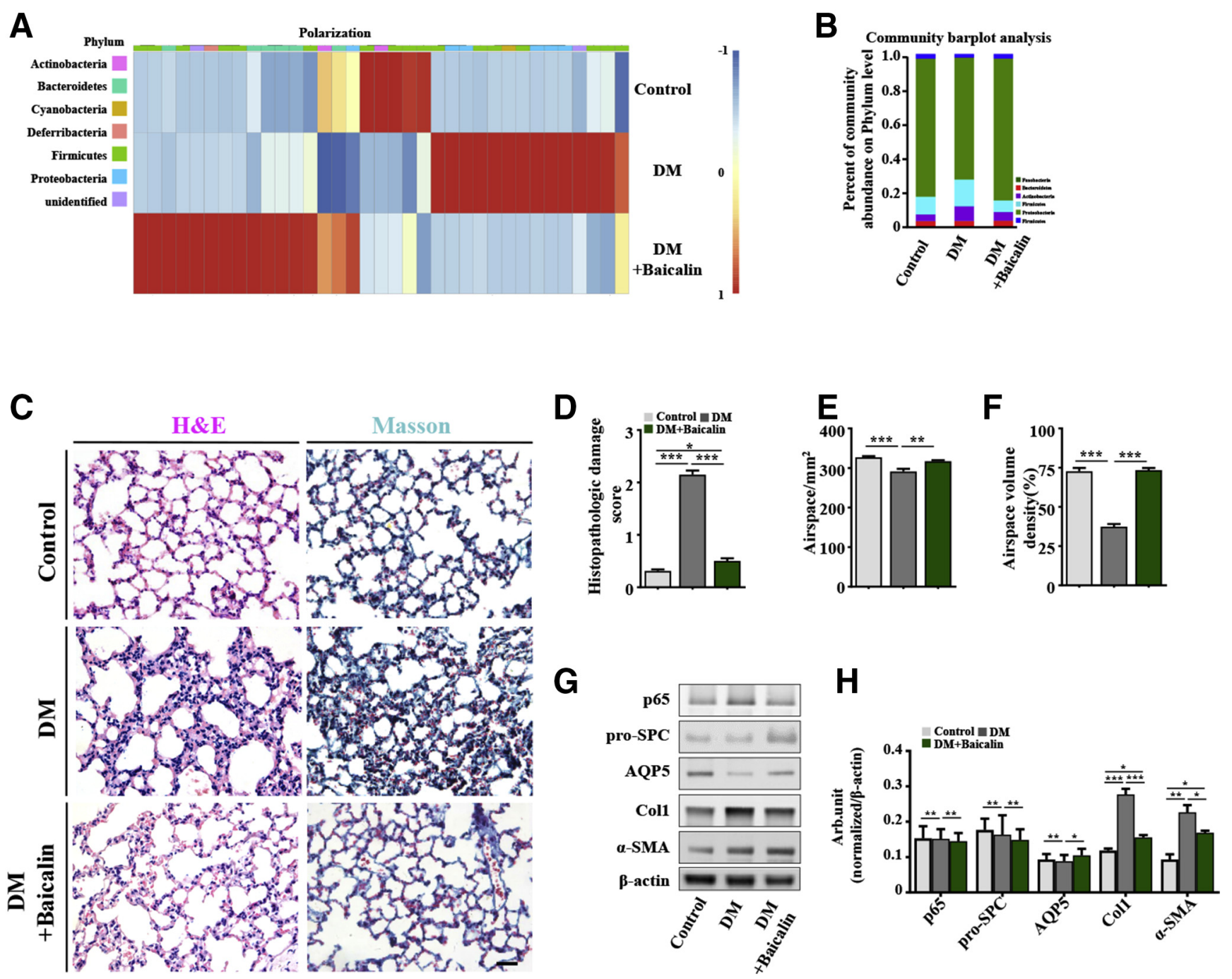

Figure 8 Assessing lung injury, NF- $\mathrm{KB}$ signaling, and the impact of pulmonary cell types in mice while successfully correcting gut dysbiosis after the oral administration of baicalin. A and B: Heat map of the differentially expressed genes in the lung microbiota in an RNA-sequencing data set (A), and the bar chart showing the comparison of the relative abundances of various bacterial divisions in the lung microbiota (B) from the control, diabetes mellitus (DM), and DM + baicalin groups. C-F: Hematoxylin and eosin (H\&E) and Masson staining on the transverse sections of mouse lungs from the control, DM, and DM + baicalin groups. D-F: The bar charts correspondingly show the histopathologic damage score (D), airspace size per millimeter (E), and percentage of airspace volume density (F). G and H: Western blot data showing p65, pro-surfactant protein C, aquaporin-5 (AQP5), collagen I (Col1), and $\alpha$-smooth muscle actin ( $\alpha$-SMA) expression in the mouse lung tissues. The bar chart shows the ratios of the band arbitrary units of the corresponding gene expression normalized to $\beta$-actin. $n=5$ (D-F); $n=3(\mathbf{H}) .{ }^{*} P<0.05, * * P<0.01$, and ${ }^{* * *} P<0.001$. Scale bars $=50 \mu \mathrm{m}(\mathbf{C})$.

indicating the increased cell death in the pulmonary alveolar epithelium or the proportionally enhanced alveolar epithelial cell mesenchymal transition accompanied by the proliferation of pulmonary interstitial cells through the activation of LOX-1/TGF- $\beta 1 / K L F 6$ signaling. ${ }^{74}$
Inflammation and the toll-like receptor $4 / \mathrm{NF}-\kappa \mathrm{B}$ signaling pathways are closely involved in diabetic lung injury, ${ }^{7,74}$ whereas DM is almost always accompanied with the dysbiosis of the gut and lung microbiome. This raised the question whether DM and the subsequent gut-

Figure 7 Assessing pulmonary fibrosis, apoptosis, and NF- $\mathrm{BB}$ signaling in diabetic mice while successfully correcting gut dysbiosis using the fecal microbiota transplant (FMT) approach. A-C: The Sirius red staining showing the higher expressions of total collagen, collagen type 1 (red or yellow), and collagen type 3 (green) in diabetes mellitus (DM) group. A and B: The bar charts showing the percentage area of total collagen, collagen type 1, and collagen type 3 in both control and DM groups. C: The bar charts showing the data of quantitative PCR for the expressions of col1a1, col3a1, and TGF- $\beta$ at mRNA level in both control and DM groups. D and E: The p65 immunofluorescence staining on the transverse sections of mouse lungs from the control, DM, and FMT groups. All of the sections were counterstained with Hoechst. White boxed areas in $\mathbf{D}$ indicate positive cells (insets). E: The chart correspondingly showing the aforementioned marker-positive cells in the total cell populations. F: Western blot data showing factor associated suicide, p53, and pro-surfactant protein C expression in the mouse lung tissues. G: The bar chart shows the ratios of the band arbitrary units of the corresponding gene expression normalized to $\beta$-actin. H: The bar chart showing the data of quantitative PCR for the expressions of Casp8, Casp9, Bcl2, and Bax. I: Western blot data showing p65 and p50 expression in the mouse lung tissues. J and K: The bar charts showing the ratios of the band arbitrary units of the corresponding gene expression normalized to $\beta$-actin $(\mathbf{J})$ and the data of quantitative PCR for the expressions of $p 65$ and $p 50$ at mRNA level in control, DM, and FMT groups, relative to $\beta$-actin mRNA level $(\mathbf{K}) . n=8(\mathbf{B}) ; n=3(\mathbf{C}, \mathbf{G}, \mathbf{H}, \mathbf{J}$, and $\mathbf{K}) ; n=18$ for control and LH + FMT (E); $n=17$ for LH (E). ${ }^{*} P<0.05,{ }^{*} P<0.01$, and ${ }^{* *} P<0.001$. Scale bars: $10 \mu \mathrm{m}(\mathbf{A}) ; 50 \mu \mathrm{m}$ (D). 
A
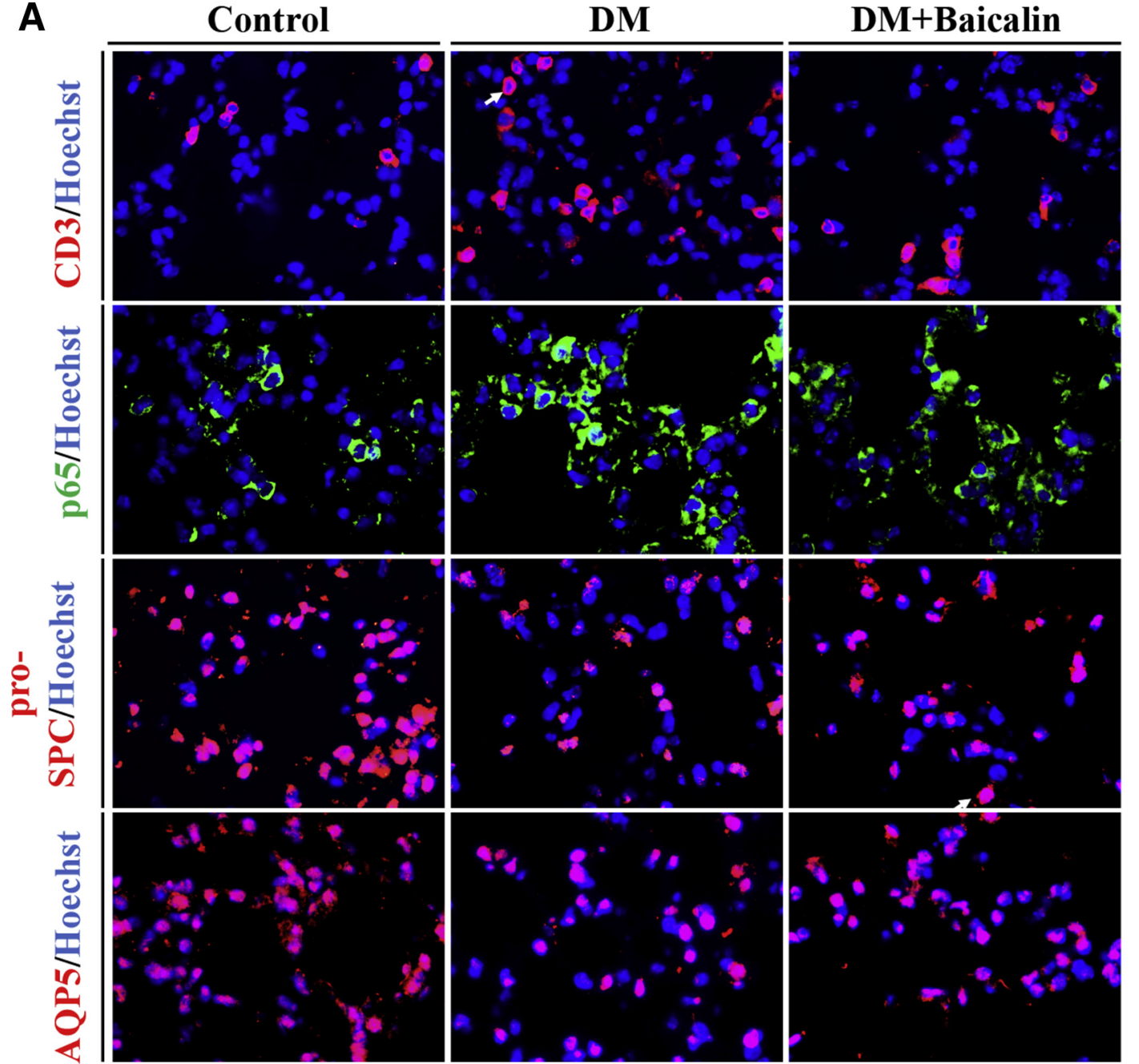

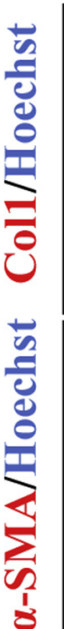
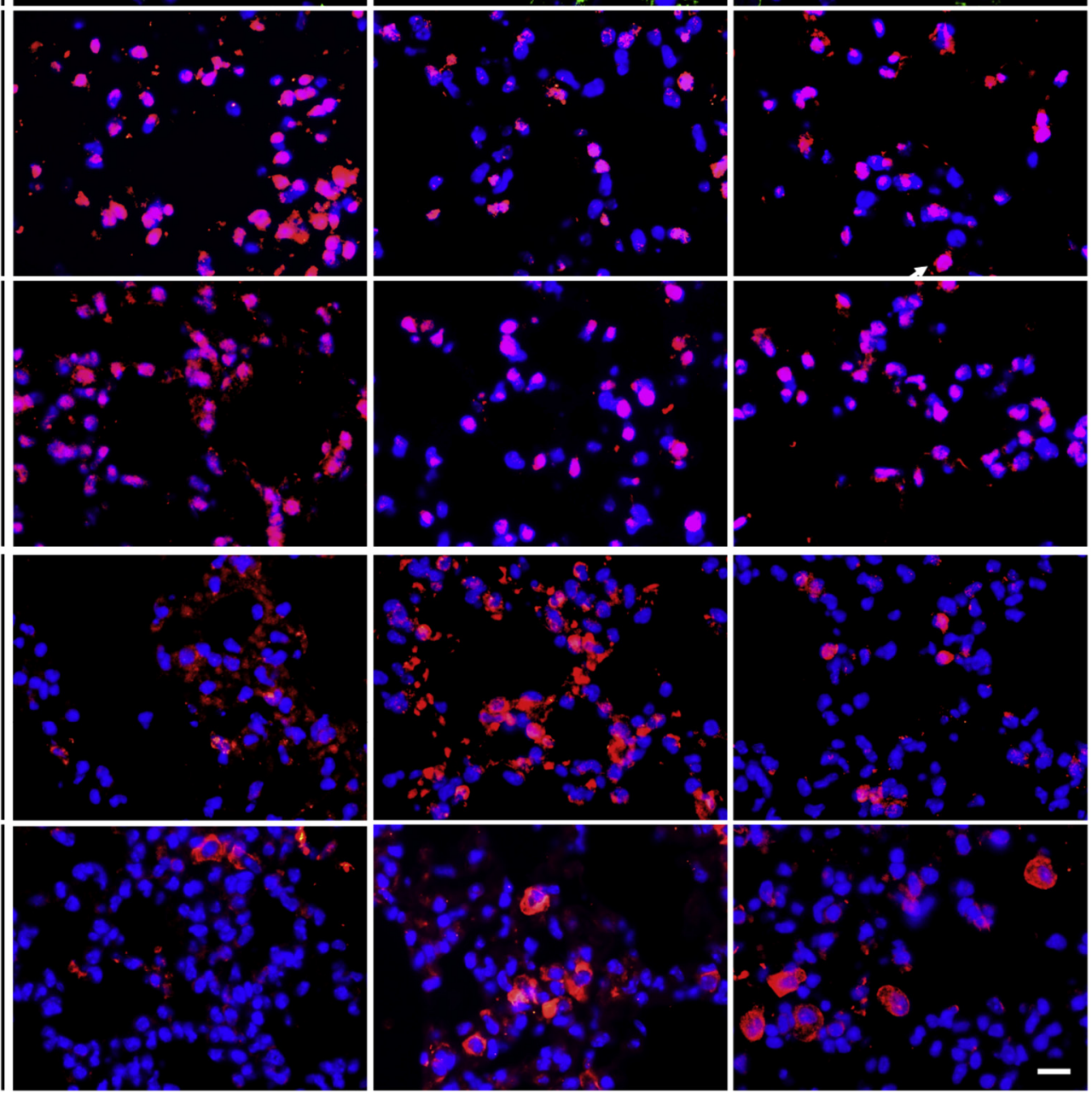

D

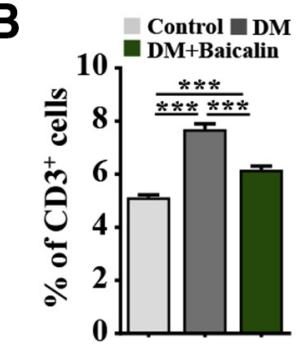

C
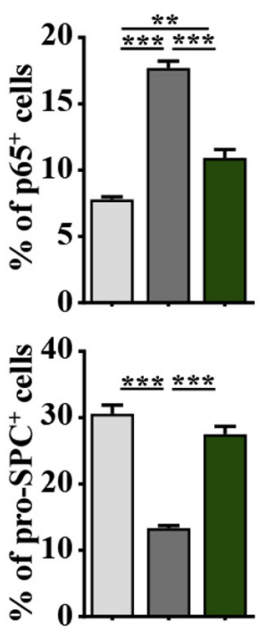

$\mathbf{E}$

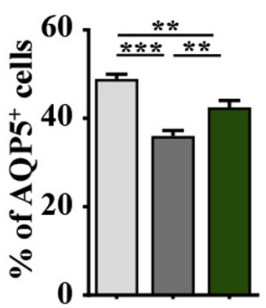

$\mathbf{F}$

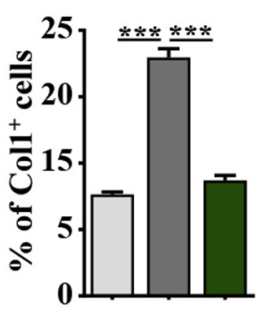

G

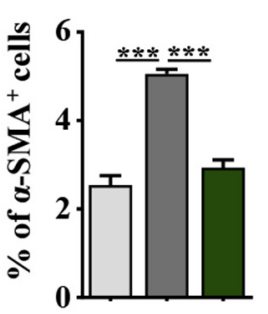

Figure 9 Assessing lung injury, NF- $\mathrm{KB}$ signaling, and the impact of pulmonary cell types in mice while successfully correcting gut dysbiosis after the oral administration of baicalin. A: CD3, p65, pro-surfactant protein C, aquaporin-5 (AQP5), collagen I (Col1), and $\alpha$-smooth muscle actin ( $\alpha$-SMA) immunofluorescence staining on the transverse sections of mouse lungs from the control, diabetes mellitus (DM), and DM + baicalin groups. All of the sections were counterstained with Hoechst. B-G: The bar charts correspondingly showing the aforementioned marker-positive cells in the total cell populations. $n=6(\mathbf{B}-\mathbf{G}) .{ }^{* *} P<0.01,{ }^{* * *} P<0.001$. Scale bars $=10 \mu \mathrm{m}(\mathbf{A})$.

lung microbiota dysbiosis activate NF- $\kappa \mathrm{B}$ signaling in a timely, well-sequenced, and coordinated manner and further lead to pulmonary lung injury. The answer seems to be in the affirmative, as indicated by the higher expression of NF- $\kappa \mathrm{B}$ signaling cascades in mice with both diabetes and gut dysbiosis, and the pulmonary fibrotic changes can be observed in the lungs of transgenic mice expressing a constitutively active NF- $\kappa \mathrm{B}$ mutant. 


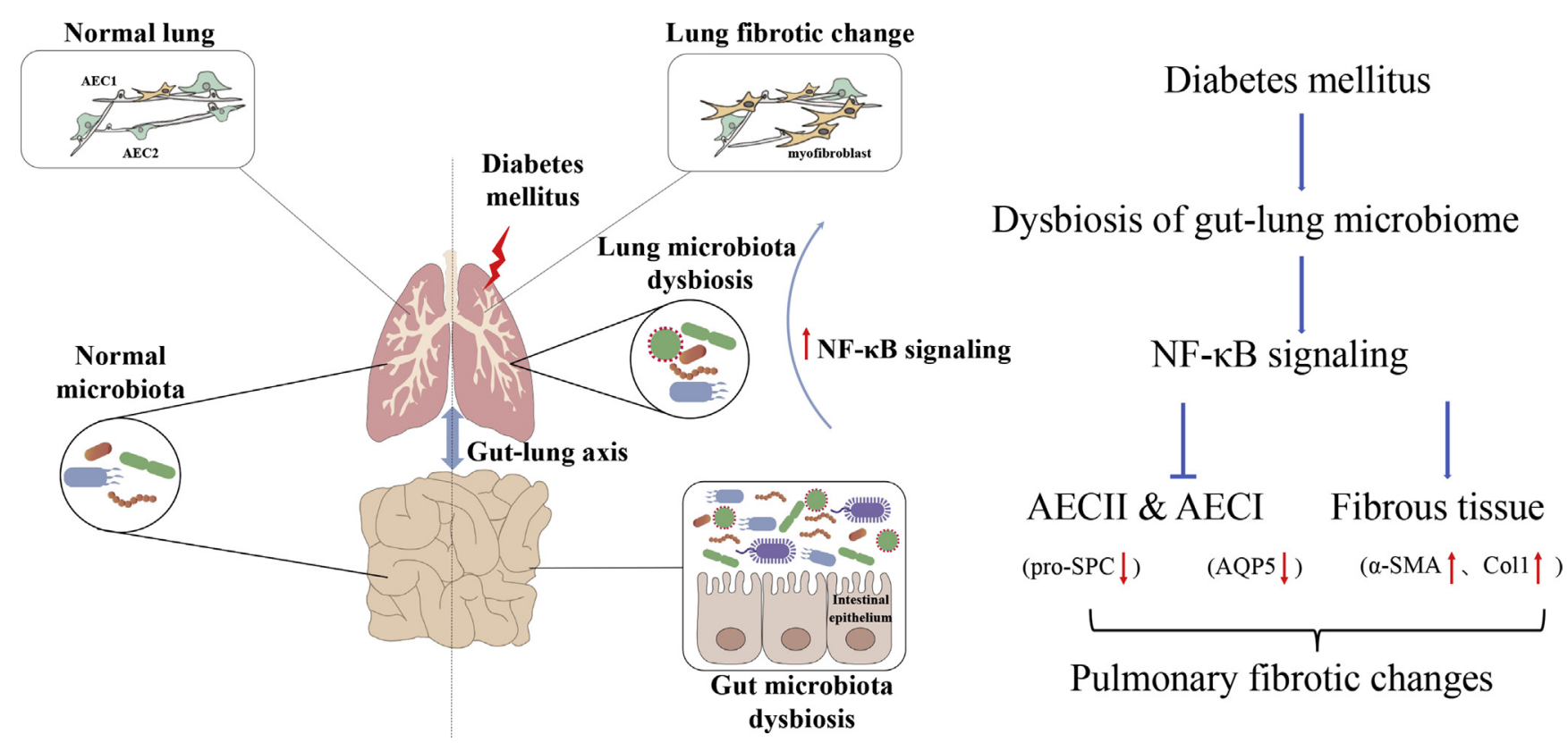

Figure 10 A proposed model that depicts the potential mechanisms for gut-lung dysbiosis accompanied by diabetes mellitus, leading to a pulmonary fibrotic change through the NF-KB signaling pathway. The left side of the dotted line is the lung and intestinal flora under the normal conditions, and the right side of the dotted line is the lung and intestinal flora under the context of diabetes. AECI, type I alveolar epithelial cell; AECII, type II alveolar epithelial cell; AQP5, aquaporin-5; Col1, collagen I; $\alpha$-SMA, $\alpha$-smooth muscle actin.

Abundant evidence supports this notion (eg, commensal and probiotic bacteria effectively reduced the inflammatory response mediated by the NF- $\kappa \mathrm{B}$ signaling pathway in the intestinal mucosa). ${ }^{75}$ Using the same strategy, the current study implemented FMT to reestablish healthy intestinal flora in the mice with gut dysbiosis induced by LH gavage and STZ-induced diabetic mice. Although FMT implementation did not reduce the mouse blood glucose in diabetic mice, it redressed the gut dysbiosis characterized by the gut microbiome returning to normal, the pulmonary fibrotic changes, increased atosis, activation of NF- $\kappa \mathrm{B}$ signaling, and enhanced inflammatory cytokine production in both LH- and DM-induced intestinal flora disorder mouse model were successfully restricted. These research data, verified with a solid evidence base, indicated that the gut and lung microbiota played indispensable roles in maintaining and regulating physiological immune responses. On the other hand, dysfunction of the gut and lung microbiota causes the onset, exacerbation, progression, and even treatment effect of lung diseases via an immunologic link between them (ie, gut-lung axis). The underlying mechanism of the bidirectional connection between the gut and lungs remains obscure, communication with each other through both systemic immune responses and the mucosal immune system. $^{76,77}$

Despite the relationship between microbial dysbiosis of the gut and lungs described above, the data still do not completely explain, the pathophysiological mechanism of diabetic lung injury. For example, Wu et al ${ }^{55}$ reported that diabetic lung injury could develop from the oxidation- reduction imbalance, mitochondrial abnormality, and oxidative stress induced by DM. Prior research also shows that baicalin administration can attenuate diabetes mellitus-induced damage of kidney and liver to some extent; and successfully reduce the blood glucose levels of SYTZ-induced diabetic mice. ${ }^{30,42}$ Hence, in this study, the treatment of diabetic mice with baicalin, with its antioxidation, anti-inflammation, and anti-apoptosis biological activities, ${ }^{53}$ led to microbial dysbiosis of the gut and lungs, activation of the NF- $\kappa \mathrm{B}$ signaling pathway, and dramatic improvement of pulmonary fibrotic changes. It is speculated that the therapeutic effect of baicalin benefited from its biological activity, implying that the pathogenesis of diabetic lung injury may be caused by comprehensive factors in which microbial dysbiosis of the gut and lungs accompanied by $\mathrm{DM}$ is in a key position and involves regulation of the toll-like receptor $4 / \mathrm{NF}-\kappa \mathrm{B}$ signaling pathway.

Data from the current study indicate that DM causes gut microbial dysbiosis, which may further affect the lung flora through the gut-lung axis (Figure 10). Subsequently microbial dysbiosis activates the NF- $\kappa$ B signaling pathway and results in a reduction in alveolar epithelial cells, promotes the production of fibrous interstitial tissue, and finally leads to fibrous changes in lung tissue.

\section{Author Contributions}

G.W. analyzed data and cowrote the manuscript; Y.-X.H. collected and analyzed data and prepared figures for the 
manuscript; M.-Y.H. analyzed data, edited the manuscript, and prepared figures for the manuscript; Y.-H.X., W.S., and D-L.L. collected data and edited the manuscript; R.Z., JY.W, Y.d., H.L., and P.S. collected data; X.C. and Z-J.L. reviewed/edited the manuscript; X.Y. managed the project, analyzed data, and edited the manuscript.

\section{Supplemental Data}

Supplemental material for this article can be found at http://doi.org/10.1016/j.ajpath.2021.02.019.

\section{References}

1. Bhutani J, Bhutani S: Worldwide burden of diabetes. Indian J Endocrinol Metab 2014, 18:868

2. American Diabetes Association: Diagnosis and classification of diabetes mellitus. Diabetes Care 2010, 33:S62-S69

3. Altobelli E, Petrocelli R, Verrotti A, Chiarelli F, Marziliano C: Genetic and environmental factors affect the onset of type 1 diabetes mellitus. Pediatr Diabetes 2016, 17:559-566

4. Murea M, Ma L, Freedman BI: Genetic and environmental factors associated with type 2 diabetes and diabetic vascular complications. Rev Diabet Stud 2012, 9:6

5. Nathan DM: Long-term complications of diabetes mellitus. N Engl J Med 1993, 328:1676-1685

6. Talakatta G, Sarikhani M, Muhamed J, Dhanya K, Somashekar BS, Mahesh PA, Sundaresan N, Ravindra P: Diabetes induces fibrotic changes in the lung through the activation of TGF- $\beta$ signaling pathways. Sci Rep 2018, 8:1-15

7. Pitocco D, Fuso L, Conte EG, Zaccardi F, Condoluci C, Scavone G, Incalzi RA, Ghirlanda G: The diabetic lung-a new target organ? the review of diabetic studies. Rev Diabet Stud 2012, 9:23

8. Tai N, Wong FS, Wen L: The role of gut microbiota in the development of type 1, type 2 diabetes mellitus and obesity. Rev Endocr Metab Disord 2015, 16:55-65

9. Alkanani AK, Hara N, Lien E, Ir D, Kotter CV, Robertson CE, Wagner BD, Frank DN, Zipris D: Induction of diabetes in the RIPB7. 1 mouse model is critically dependent on TLR3 and MyD88 pathways and is associated with alterations in the intestinal microbiome. Diabetes 2014, 63:619-631

10. Sato J, Kanazawa A, Ikeda F, Yoshihara T, Goto H, Abe H, Komiya K, Kawaguchi M, Shimizu T, Ogihara T: Gut dysbiosis and detection of "live gut bacteria" in blood of Japanese patients with type 2 diabetes. Diabetes Care 2014, 37:2343-2350

11. Ringel-Kulka T, Cheng J, Ringel Y, Salojärvi J, Carroll I, Palva A, de Vos WM, Satokari R: Intestinal microbiota in healthy US young children and adults - a high throughput microarray analysis. PLoS One 2013, 8:e64315

12. Tilg H, Moschen AR: Microbiota and diabetes: an evolving relationship. Gut 2014, 63:1513-1521

13. Qin J, Li Y, Cai Z, Li S, Zhu J, Zhang F, Liang S, Zhang W, Guan Y, Shen D: A metagenome-wide association study of gut microbiota in type 2 diabetes. Nature 2012, 490:55-60

14. He Y, Wen Q, Yao F, Xu D, Huang Y, Wang J: Gut-lung axis: the microbial contributions and clinical implications. Crit Rev Microbiol 2017, 43:81-95

15. Huang YJ, Boushey HA: The microbiome in asthma. J Allergy Clin Immunol 2015, 135:25-30

16. Lee Y-T, Kim K-H, Hwang HS, Lee Y, Kwon Y-M, Ko E-J, Jung YJ, Lee Y-N, Kim M-C, Kang S-M: Innate and adaptive cellular phenotypes contributing to pulmonary disease in mice after respiratory syncytial virus immunization and infection. Virology 2015, 485:36-46

17. Segal LN, Blaser MJ: A brave new world: the lung microbiota in an era of change. Ann Am Thorac Soc 2014, 11:S21-S27

18. Erb-Downward JR, Huffnagle GB, Martinez FJ: The Microbiota in Respiratory Disease. Ann Arbor, MI: American Thoracic Society, 2012

19. Zaph C, Troy AE, Taylor BC, Berman-Booty LD, Guild KJ, Du Y, Yost EA, Gruber AD, May MJ, Greten FR: Epithelial-cell-intrinsic IKK- $\beta$ expression regulates intestinal immune homeostasis. Nature 2007, 446:552-556

20. Oeckinghaus A, Ghosh $\mathrm{S}$ : The NF- $\mathrm{kB}$ family of transcription factors and its regulation. Cold Spring Harb Perspect Biol 2009, 1:a000034

21. Baker RG, Hayden MS, Ghosh S: NF- $\mathrm{B}$, inflammation, and metabolic disease. Cell Metab 2011, 13:11-22

22. Toledano M, Ghosh D, Trinh F, Leonard W: N-terminal DNAbinding domains contribute to differential DNA-binding specificities of NF-kappa B p50 and p65. Mol Cell Biol 1993, 13: $852-860$

23. Xu JJ, Wang G, Luo X, Wang LJ, Bao Y, Yang X: Role of nuclear factorins contribute to differential DNA-binding specificities. J Cell Physiol 2019, 234:22565-22580

24. Kumar SD, Dheen ST, Tay SSW: Maternal diabetes induces congenital heart defects in mice by altering the expression of genes involved in cardiovascular development. Cardiovasc Diabetol 2007 $6: 34$

25. Wang LM, Zhong NZ, Liu SJ, Zhu XY, Liu YJ: Hypoxia-induced acute lung injury is aggravated in streptozotocin diabetic mice. Exp Lung Res 2015, 41:146-154

26. Yuan $\mathrm{D}$, Xu Y, Hang $\mathrm{H}$, Liu $\mathrm{X}$, Chen $\mathrm{X}$, Xie $\mathrm{P}$, Yuan $\mathrm{S}$, Zhang W, Lin X, Liu Q: Edaravone protect against retina damage in streptozotocin-induced diabetic mice. PLoS One 2014, 9:e99219

27. Jin Y, Wang G, Han SS, He MY, Cheng X, Ma ZL, Wu X, Yang X, Liu GS: Effects of oxidative stress on hyperglycaemia-induced brain malformations in a diabetes mouse model. Exp Cell Res 2016, 347 201-211

28. He MY, Wang G, Han SS, Jin Y, Li H, Wu X, Ma ZL, Cheng X, Tang X, Yang X, Liu GS: Nrf2 signalling and autophagy are involved in diabetes mellitus-induced defects in the development of mouse placenta. Open Biol 2016, 6:160064

29. Han SS, Wang G, Jin Y, Ma ZL, Jia WJ, Wu X, Wang XY, He MY, Cheng X, Li WJ, Yang X, Liu GS: Investigating the mechanism of hyperglycemia-induced fetal cardiac hypertrophy. PLoS One 2015 10:e139141

30. Wang G, Liang J, Gao LR, Si ZP, Zhang XT, Liang G, Yan Y, Li K, Cheng X, Bao Y, Chuai M, Chen LG, Lu DX, Yang X: Baicalin administration attenuates hyperglycemia-induced malformation of cardiovascular system. Cell Death Dis 2018, 9:234

31. Si ZP, Wang G, Han SS, Jin Y, Hu YX, He MY, BrandSaberi B, Yang X, Liu GS: CNTF and Nrf2 are coordinately involved in regulating self-renewal and differentiation of neural stem cell during embryonic development. iScience 2019, 19: $303-315$

32. Liu J, Wu M, He J, Xiao C, Xue Y, Fu T, Lin C, Dong D, Li Z Antibiotic-induced dysbiosis of gut microbiota impairs corneal nerve regeneration by affecting CCR2-negative macrophage distribution. Am J Pathol 2018, 188:2786-2799

33. Song J, Wang C, Long D, Li Z, You L, Brand-Saberi B, Wang G, Yang X: Dysbacteriosis-induced LPS elevation disturbs the development of muscle progenitor cells by interfering with retinoic acid signaling. FASEB J 2020, 34:6837-6853

34. Suez J, Korem T, Zeevi D, Zilberman-Schapira G, Thaiss CA, Maza O, Israeli D, Zmora N, Gilad S, Weinberger A, Kuperman Y, Harmelin A, Kolodkin-Gal I, Shapiro H, Halpern Z, Segal E, Elinav E: Artificial sweeteners induce glucose intolerance by altering the gut microbiota. Nature 2014, 514:181-186 
35. Lattouf R, Younes R, Lutomski D, Naaman N, Godeau G, Senni K, Changotade S: Picrosirius red staining: a useful tool to appraise collagen networks in normal and pathological tissues. J Histochem Cytochem 2014, 62:751-758

36. Wang G, Yeung CK, Wong WY, Zhang N, Wei YF, Zhang JL, Yan Y, Wong CY, Tang JJ, Chuai ML, Lee KKH, Wang LJ, Yang XS: Liver fibrosis can be induced by high salt intake through excess reactive oxygen species (ROS) production. J Agric Food Chem 2016, 64:1610-1617

37. Kauffman SL: Acceleration of canalicular development in lungs of fetal mice exposed transplacentally to dexamethasone. Lab Invest 1977, 36:395-401

38. Plosa EJ, Young LR, Gulleman PM, Polosukhin VV, Zaynagetdinov R, Benjamin JT, Im AM, van der Meer R, Gleaves LA, Bulus N, Han W, Prince LS, Blackwell TS, Zent R: Epithelial betal integrin is required for lung branching morphogenesis and alveolarization. Development 2014, 141: $4751-4762$

39. Zhang J, Wang G, Liu J, Gao LR, Liu M, Wang CJ, Chuai M, Bao Y, Li G, Li RM, Zhang Y, Yang X: Gut microbiota-derived endotoxin enhanced the incidence of cardia bifida during cardiogenesis. J Cell Physiol 2018, 233:9271-9283

40. Xu JJ, Wang G, Luo X, Wang LJ, Bao Y, Yang X: Role of nuclear factor-kappaB pathway in the transition of mouse secondary follicles to antral follicles. J Cell Physiol 2019, 234: 22565-22580

41. He MY, Wang G, Han SS, Li K, Jin Y, Liu M, Si ZP, Wang J, Liu GS, Yang X: Negative impact of hyperglycaemia on mouse alveolar development. Cell Cycle 2018, 17:80-91

42. Zhang XT, Wang G, Ye LF, Pu Y, Li RT, Liang J, Wang L, Lee KKH, Yang X: Baicalin reversal of DNA hypermethylationassociated Klotho suppression ameliorates renal injury in type 1 diabetic mouse model. Cell Cycle 2020, 19:3329-3347

43. Paudel KR, Kim DW: Microparticles-mediated vascular inflammation and its amelioration by antioxidant activity of baicalin. Antioxidants (Basel) 2020, 9:890

44. Wang G, Lu JQ, Ding Y, Zhang T, Song JH, Long D, Liang J, Cheng X, Si Z, Qi G, Jiang X, Yang X: Baicalin rescues hyperglycemia-induced neural tube defects via targeting on retinoic acid signaling. Am J Transl Res 2020, 12:3311-3328

45. Mulliken JB, Burrows PE, Fishman SJ: Mulliken and Young's Vascular Anomalies: Hemangiomas and Malformations: Oxford University Press; 2013

46. Flodby P, Li C, Liu Y, Wang H, Rieger ME, Minoo P, Crandall ED, Ann DK, Borok Z, Zhou B: Cell-specific expression of aquaporin-5 (Aqp5) in alveolar epithelium is directed by GATA6/Sp1 via histone acetylation. Sci Rep 2017, 7:1-12

47. Kim KK, Kugler MC, Wolters PJ, Robillard L, Galvez MG, Brumwell AN, Sheppard D, Chapman HA: Alveolar epithelial cell mesenchymal transition develops in vivo during pulmonary fibrosis and is regulated by the extracellular matrix. Proc Natl Acad Sci U S A 2006, 103:13180-13185

48. Bringardner BD, Baran CP, Eubank TD, Marsh CB: The role of inflammation in the pathogenesis of idiopathic pulmonary fibrosis. Antioxid Redox Signal 2008, 10:287-301

49. Hou J, Ma T, Cao H, Chen Y, Wang C, Chen X, Xiang Z, Han X: TNF- $\alpha$-induced NF- $\kappa B$ activation promotes myofibroblast differentiation of LR-MSCs and exacerbates bleomycin-induced pulmonary fibrosis. J Cell Physiol 2018, 233:2409-2419

50. Magness ST, Jijon H, Fisher NVH, Sharpless NE, Brenner DA, Jobin C: In vivo pattern of lipopolysaccharide and anti-CD3-induced NF- $\kappa \mathrm{B}$ activation using a novel gene-targeted enhanced GFP reporter gene mouse. J Immunol 2004, 173:1561-1570

51. Drakopanagiotakis F, Xifteri A, Polychronopoulos V, Bouros D: Apoptosis in lung injury and fibrosis. Eur Respir J 2008, 32: $1631-1638$
52. Fernandez IE, Eickelberg O: The impact of TGF-beta on lung fibrosis: from targeting to biomarkers. Proc Am Thorac Soc 2012, 9: $111-116$

53. Yan S, Wang $\mathrm{Y}$, Liu $\mathrm{P}$, Chen $\mathrm{A}$, Chen $\mathrm{M}$, Yao D, Xu X, Wang L, Huang X: Baicalin attenuates hypoxia-induced pulmonary arterial hypertension to improve hypoxic cor pulmonale by reducing the activity of the p38 MAPK signaling pathway and MMP-9. Evid Based Complement Alternat Med 2016, 2016: 2546402

54. Wang XM, Zhang Y, Kim HP, Zhou Z, Feghali-Bostwick CA, Liu F, Ifedigbo E, Xu X, Oury TD, Kaminski N, Choi AM: Caveolin-1: a critical regulator of lung fibrosis in idiopathic pulmonary fibrosis. $\mathrm{J}$ Exp Med 2006, 203:2895-2906

55. Wu J, Jin Z, Yan L-J: Redox imbalance and mitochondrial abnormalities in the diabetic lung. Redox Biol 2017, 11:51-59

56. Vracko R, Thorning D, Huang TW: Basal lamina of alveolar epithelium and capillaries: quantitative changes with aging and in diabetes mellitus. Am Rev Respir Dis 1979, 120:973-983

57. Weynand B, Jonckheere A, Frans A, Rahier J: Diabetes mellitus induces a thickening of the pulmonary basal lamina. Respiration 1999. 66:14-19

58. Matsubara T: A study on pulmonary dysfunction and peripheral lung tissue lesions in human diabetes. J Nippon Med Sch 1991, 58: $528-536$

59. Fariña J, Furió V, Fernandez-Aceñero MJ, Muzas MA: Nodular fibrosis of the lung in diabetes mellitus. Virchows Archiv 1995, 427: 61-63

60. Ban CR, Twigg SM: Fibrosis in diabetes complications: pathogenic mechanisms and circulating and urinary markers. Vasc Health Risk Manag 2008, 4:575

61. Wynn TA, Ramalingam TR: Mechanisms of fibrosis: therapeutic translation for fibrotic disease. Nat Med 2012, 18:1028

62. Chakradhar S: A curious connection: teasing apart the link between gut microbes and lung disease. Nat Med 2017, 23: 402-404

63. Huang X, Fan X, Ying J, Chen S: Emerging trends and research foci in gastrointestinal microbiome. J Transl Med 2019, 17:67

64. Enaud R, Prevel R, Ciarlo E, Beaufils F, Wieërs G, Guery B, Delhaes L: The gut-lung axis in health and respiratory diseases: a place for inter-organ and inter-kingdom crosstalks. Front Cell Infect Microbiol 2020, 10:9

65. Baral V, Connett G: Acute intestinal obstruction as a presentation of cystic fibrosis in infancy. J Cyst Fibros 2008, 7:277-279

66. Roussos A, Koursarakos P, Patsopoulos D, Gerogianni I, Philippou N: Increased prevalence of irritable bowel syndrome in patients with bronchial asthma. Respir Med 2003, 97:75-79

67. Keely S, Hansbro PM: Lung-gut cross talk: a potential mechanism for intestinal dysfunction in patients with COPD. Chest 2014, 145: $199-200$

68. Wang J, Li F, Wei H, Lian Z-X, Sun R, Tian Z: Respiratory influenza virus infection induces intestinal immune injury via microbiotamediated Th17 cell-dependent inflammation. J Exp Med 2014, 211: $2397-2410$

69. Dilantika C, Sedyaningsih ER, Kasper MR, Agtini M, Listiyaningsih E, Uyeki TM, Burgess TH, Blair PJ, Putnam SD: Influenza virus infection among pediatric patients reporting diarrhea and influenza-like illness. BMC Infect Dis 2010, 10:3

70. Zhang Z-M, Wang Y-C, Chen L, Li Z: Protective effects of the suppressed NF-KB/TLR4 signaling pathway on oxidative stress of lung tissue in rat with acute lung injury. Kaohsiung J Med Sci 2019, $35: 265-276$

71. Tang J, Xu L, Zeng Y, Gong F: Effect of gut microbiota on LPSinduced acute lung injury by regulating the TLR4/NF-kB signaling pathway. Int Immunopharmacol 2020, 91:107272

72. Zheng H, Wu J, Jin Z, Yan L-J: Potential biochemical mechanisms of lung injury in diabetes. Aging Dis 2017, 8:7 
73. Alkan M, Çelik A, Bilge M, Kiraz HA, Kip G, Özer A, Şıvgın V Erdem Ö, Arslan M, Kavutçu M: The effect of levosimendan on lung damage after myocardial ischemia reperfusion in rats in which experimental diabetes was induced. J Surg Res 2015, 193: 920-925

74. Zou X-Z, Gong Z-C, Liu T, He F, Zhu T-T, Li D, Zhang W-F, Jiang J-L, Hu C-P: Involvement of epithelial-mesenchymal transition afforded by activation of LOX-1/TGF- $\beta 1 /$ KLF6 signaling pathway in diabetic pulmonary fibrosis. Pulm Pharmacol Ther 2017, 44:70-77
75. Kaci G, Lakhdari O, Doré J, Ehrlich SD, Renault P, Blottière HM, Delorme C: Inhibition of the NF- $\kappa \mathrm{B}$ pathway in human intestinal epithelial cells by commensal Streptococcus salivarius. Appl Environ Microbiol 2011, 77:4681-4684

76. Cesta MF: Normal structure, function, and histology of mucosa-associated lymphoid tissue. Toxicol Pathol 2006, 34: 599-608

77. Barcik W, Boutin RC, Sokolowska M, Finlay BB: The role of lung and gut microbiota in the pathology of asthma. Immunity 2020, 52: $241-255$ 